\title{
Adressierung in der ein- und zwei- sprachigen Lexikographie. Eine einführende Übersicht über die Forschungs- und Problemlage
}

\section{Herbert Ernst Wiegand, Germanistisches Seminar, Universität Heidelberg, Heidelberg, Bundesrepublik Deutschland}

Zusammenfassung: Obwohl die Adressierungsbeziehung eine wichtige textuelle Beziehung insbesondere in Wörterbuchartikeln ist, ohne deren theoretisches Verständnis die Gewinnung von lexikographischen Informationen anhand von hochverdichteten und stark standardisierten Wörterbuchartikeln nicht erklärt werden kann, gibt es bisher nur werige Beiträge, in denen die Adressierung theoriebezogen behandelt wurde.

Zunächst werden einige theoretische Voraussetzungen aus einer Theorie der Wörterbuchform eingeführt. Erläutert wird erstens die Standardisierung an Beispielen zur lexikographischen Beschreibungssprache, anhand der inneren Textverdichtung, an Beispielen zum Wörterbuchgegenstand und bezüglich der textuellen Strukturen von Wörterbuchartikeln. Daraufhin wird zweitens gezeigt, wie man sich den Prozeß der Textverdichtung als Textverarbeitungsprozeß denken kann, der von einem Volltext zu einem verdichteten Artikeltext führt; es wird deutlich, daß hierbei die syntaktische Struktur des Volltextes weitgehend getilgt wird, so daß im Artikeltext keine Kohäsionsanweisungen und syntaktischen Funktionen erkennbar sind. Damit die syntaktischen Konstituenten (die Angaben) aufeinander bezogen werden können, müssen die Angaben an andere Angaben adressiert sein. Adressierungsbeziehungen sind daher der Ersatz für die syntaktischen Funktionen und kohäsiven Bezüge. Schließlich werden drittens Textkonstituentenstrukturen eingeführt, und zwar wird insbesondere dargelegt, wie Mikrostrukturen zu analysieren sind.

Auf der Basis der eingeführten Voraussetzungen werden dann zuerst einsprachige Wörterbuchartikel hinsichtlich ihrer Adressierungsstrukturen exemplarisch untersucht. Dabei werden verschiedene Arten der artikelintemen Adressierung unterschieden, z.B. lemmatische, vollständig lemmatische, sublemmatische, nichtlemmatische, standardisierte, nichtstandardisierte Adressierung sowie Links- und Rechtsadressierung und einige weitere Arten. Weiterhin wird dargelegt, $\mathrm{da} ß$ die Adrressierungsrelation in Wörterbuchartikeln mit vollständiger lemmatischer Adressierung eine Funktion ist und wie die Angabestruktur eines Wörterbuchartikels als Zusammenfügung von Mikro- und Adressierungsstruktur erklärt und dargestellt werden kann.

Schließlich wird im letzten Abschnitt auf Aspekte der beiden wichtigsten Adressierungsprobleme in zweisprachigen Wörterbuchartikeln eingegangen. Diese entstehen (i) dadurch, daß die Äquivalentbeziehung meistens eine bedingte Beziehung ist, die nur gilt, wenn andere Sachverhalte gelten, und (ii) weiterhin dadurch, daß es zu einem ausgangssprachlichen Lemmazeichen mehrere zielsprachliche Äquivalente geben kann. Dadurch werden Äquivalentunterscheidungsangaben 
nötig, mit denen komplexe Adressierungen notwendig werden. Es wird vorgeschlagen, diese als lexikographisch vertextete wenn-dann-Beziehungen zu verstehen und entsprechend in den Benutzungshinweisen zu erklären.

Stichwörter: ADRESSIERUNG, ADRESSE, ANGABE, WORTERBUCHARTIKEL, STANDARDISIERUNG，TEXTVERDICHTUNG，MIKROSTRUKTUR， ADRESSIERUNGSSTRUKTUR, ANGABESTRUKTUR

Abstract: Addressing in Mono- and Bilingual Lexicography. An Introduc-
tory Survey of the State of Research and Problematics. The addressing relationship is an important textual relationship, especially in dictionary articles. Without the theoretical understanding thereof, lexicographical information obtained from highly condensed and greatly standardised dictionary articles cannot be interpreted. Up to now, however, there have only been a few contributions in which addressing has been theoretically treated.

To begin with, some theoretical prerequisites from a theory of dictionary form is introduced. Firstly, standardisation is explained using examples from lexicographic descriptive language, referring to internal textual condensation, using examples from the dictionary object and referring to the textual structures of dictionary articles. Secondly, it is shown how the process of textual condensation as text processing procedure can be conceived of as leading from the full text to the condensed text of the article. It becomes clear that in this process the syntactic structure of the full text is considerably reduced, so that no indicators of cohesion and syntactic functions are recognisable in the text of the article. Items should be addressed to other items so that the syntactic constituents (the items) can be referred to one another. Addressing relationships are therefore substitutes for syntactic functions and cohesive relations. Finally, the text constituting structures are introduced, and it is explained how microstructures can be analysed.

On the basis of the introduced prerequisites, the addressing structures of monolingual dictionary articles are then firstly examined by way of example. In this process different types of article internal addressing are distinguished, e.g. lemmatic, full lemmatic, sublemmatic, nonlemmatic, standardised, nonstandardised addressing, as well as left and right addressing and several other types. It is further explained that the addressing relationship in dictionary articles with full lemmatic addressing is a function and how the entry structure of a dictionary article can be explained and presented as a junction of the micro- and addressing structure.

In the last section aspects of the two most important addressing problems in bilingual dictionaries are discussed. These originate (i) from the fact that the equivalence relationship is usually a qualified relationship which is only valid when other conditions apply, and (ii) also from the fact that one source language lemma sign may have more than one target language equivalent. This makes equivalent discrimination entries necessary, which necessitates complex addressing. It is suggested that these should be understood as lexicographically textualised if-then relationships and be explained as such in the usage notes.

Keywords: ADDRESSING, ADDRESS, ITEM, DICTIONARY ARTICLE, STANDARDISATION, TEXT CONDENSATION, MICROSTRUCTURE, ADDRESSING STRUCTURE, ITEM STRUCTURE 


\section{Zum Forschungsstand und zum theoretischen Rahmen}

Die Adressierungsbeziehung ist eine der wichtigsten textuellen Beziehungen in lexikographischen Texten mit einem Leitelementträger, insbesondere in hochverdichteten und stark standardisierten Wörterbuchartikeln in gedruckten einund zweisprachigen Sprachwörterbüchern, und zwar sowohl in tabellarischen Wörterbuchartikeln (i.S.v. Wiegand 2000d) als auch in nichttabellarischen. Grob gesprochen, wird durch Adressierungsbeziehungen der Bezug von lexikographischen Daten zu anderen lexikographischen Daten und damit zum Wörterbuchgegenstand (i.S.v. Wiegand 1998: 302, D 3-5) geregelt. Nur ein Benutzer, der in der Lage ist, z.B. eine Wörterbuchangabe (kurz: Angabe) korrekt auf eine andere Angabe zu beziehen, kann anhand der Angaben eines Wörterbuchartikels lexikographische Informationen (als kognitive Gegebenheiten) erschließen.

Trotz der zentralen Bedeutung der Adressierungsbeziehungen für das Verständnis von Teilen der Wörterbuchform und der Informationsgewinnung bei der Wörterbuchbenutzung gibt es bisher nur relativ wenige Arbeiten zur Adressierung. Die Feststellung von Hausmann und Wiegand (1989: 353): "Systematic studies of the addressing practices of dictionaries all over the world are a desideratum of dictionary research for several reasons" gilt auch heute noch. Vor dem Erscheinen des dreibändigen Handbuches zur Lexikographie (HSK 5.1-5.3) in den Jahren 1989-1991 wurde die Adressierung m.W. nicht zusammenhängend in einer eigenen Publikation thematisiert. Auch in den wichtigsten Monographien, die vor HSK 5.1-5.3 erschienen sind, wie z.B. Rey-Debove (1971), Zgusta (1971), Landau (1984), Wolski (1986) u.a., bzw. in solchen, die vorher abgeschlossen waren, wie z.B. Svensén (1993), spielt die Adressierung keine Rolle. Selbst in Übersichtswerken nach 1991, wie z.B. in Bergenholtz und Tarp (1995), wird die Adressierung nicht behandelt.

Für die Berücksichtigung der Terminologie im Bereich der Adressierung in den Wörterbüchern zur Lexikographie gilt folgendes: In Martinez de Sousa (1995) findet sich kein Lemma, das als spanisches Äquivalent zu Adresse oder Adressierung gelten kann. Auch in Burkhanov (1998) ist die Adressierung nicht berücksichtigt. In Bergenholtz et al. (1997) findet man folgende Lemmata: adresse (mit der Verweisangabe " $\rightarrow$ adressering, angivelse, bearbeidingsenhet"), adressering (mit der Verweisangabe " $\rightarrow$ adresse") und adresseringsstruktur (mit der Verweisangabe " $\rightarrow$ bearbeidingsenhet"). Die in Bergenholtz et al. (1997) begonnene lexikographische Aufarbeitung der neueren metalexikographischen Terminologie im Bereich der Adressierungsphänomene wird in Hartmann und James (1998) nicht angemessen fortgesetzt; hier wurde nur das Lemma address angesetzt. ${ }^{1}$ In den neueren linguistischen Wörterbüchern ist die metalexikographische Terminologie notorisch unterrepräsentiert; in Glück (2000) als dem deutschen sprachwissenschaftlichen Wörterbuch, das die meisten metalexikographischen Termini primär gebucht hat, findet man zwar einen Verweisartikel "Adresse $\nearrow$ Lemma”, unter dem Lemma Lemma steht aber nichts zu Adressen oder zur Adressierung. 
Seit der expliziten Behandlung der Adressierung in Hausmann und Wiegand (1989) für die einsprachige, in Hausmann/Wemer (1991) für die zweisprachige Lexikographie sowie in Wiegand (1989a) im systematischen Zusammenhang mit anderen textuellen Strukturen von Wörterbuchartikeln wurden Probleme der Adressierung nur relativ selten angesprochen. Einige Ausführungen finden sich in Wiegand (1990: 97-107) und neuerdings in Meyer und Wiegand (2000) sowie in Wiegand (2000e). Der Grund für die Zurückhaltung der Forschung bei der Bearbeitung von Fragen der Adressierung ist möglicherweise mit folgendem Zitat angesprochen: "A theoretical well-founded treatment of the addressing in the general monolingual dictionary belongs to the most difficult fields of an explicit metalexicographic description" (Wiegand 1990: 97). Für die zweisprachige Lexikographie ist eine explizite Darstellung der Adressierungsbeziehungen noch erheblich komplizierter (vgl. Wiegand 2000e); es muß m.E. derzeit sogar als offen gelten, ob die Adressierungsbeziehungen in zweisprachigen Wörterbuchartikeln in der bisherigen Forschung überhaupt hirreichend genau verstanden sind.

Eine separate ad-hoc-Behandlung der Adressierungsproblematik ohne Anbindung an eine Wörterbuchtheorie ist wenig nützlich. Im folgenden wird daher an eine weitgehend ausgearbeitete Theorie lexikographischer Textträger angeschlossen. ${ }^{2}$ Diese Theorie besteht aus zwei Teiltheorien: einer Theorie des Wörterbuchgegenstandes und einer Theorie der Wörterbuchform (vgl. Wiegand 1998: 7ff.). Im Rahmen der letztgenannten Theorie wird auch die Adressierung behandelt. Die Theorie der Wörterbuchform bildet auch für diesen Beitrag den engeren Argumentationsrahmen. Da ich eine detaillierte Kenntnis dieser Teiltheorie einer allgemeinen Theorie der Lexikographie nicht voraussetzen möchte, werden im nächsten Abschnitt zuerst einige Begrifflichkeiten informell eingeführt, die hier benötigt werden, um eine zwar einführende, aber dennoch theorieorientierte Darstellung der Adressierung geben zu können. Es sei ausdrücklich darauf hingewiesen, daß die einführenden Ausführungen im nächsten Abschnitt relativ grobmaschig sind, so daß für ein vertieftes Verständnis die jeweils genannte Literatur $\mathbf{z u}$ berücksichtigen ist.

\section{Einige theoretische Voraussetzungen}

In diesem Abschnitt werden folgende Theoriebereiche berücksichtigt, allerdings nur insoweit, als sie die Wörterbuchartikel in gedruckten Sprachwörterbüchern betreffen:

- die Standardisierung,

- die Textverdichtung, und

- die Textkonstituentenstrukturen von Wörterbuchartikeln mit Schwerpunkt auf den Mikrostrukturen. 


\subsection{Zur Standardisierung}

Eine Übersicht über die Möglichkeiten und Probleme der lexikographischen Standardisierung ist Wiegand (1997). Die Standardisierung in der Lexikographie erfolgt aufgrund von Standardisierungsinstruktionen. Diese beziehen sich auf die lexikographischen Handlungen im lexikographischen Prozeß. Sie legen fest, daß die Handlungen in bestimmter Weise anhand von Mustern, vorgegebenen Schemata, nach bestimmten Standards etc. ausgeführt werden. So werden beispielsweise Wörterbuchartikel nach vorgegebenen Musterartikeln verfaßt. Bei Wörterbuchartikeln bezieht sich die Standardisierung vor allem auf folgende artikelinterne Phänomene:

(a) die lexikographische Beschreibungssprache,

(b) die lexikographische Textverdichtung,

(c) den Artikelgegenstand als Teil des Wörterbuchgegenstandes, und

(d) die textuellen Strukturen und Architekturen des Artikels.

$Z u$ (a): Die Standardisierung der Beschreibungssprache betrifft u.a. die Ergebnisse der Textverdichtung, z.B. einheitliche Abkürzungen linguistischer Termini oder einheitliche Ersetzung der Lemmazeichen in Beispielangaben (nicht einmal durch eine Tilde " ", ein anderes mal durch einen Divis "- ${ }^{\prime \prime}$ ) u.v.a.m. Häufig wird in die Beschreibungssprache an kulturspezifische Standardisierungstraditionen in der Sprachwissenschaft angeschlossen. Beispielsweise steht in etymologischen Wörterbüchern der Asterisk mit der Bedeutung von erschlossene Form und das Zeichen " $<$ in der Bedeutung von seit (z.B. Band $n . \quad(<8$. Jh.). Mhd. bant, ahd. bant, as. band aus g. *banda- n. [...]; Kluge 1995: 77).

Auch zahlreiche wörterbuchspezifische Besonderheiten der lexikographischen Beschreibungssprache können standardisiert sein. Beispiele dafür finden sich in dem BW-Artikel in Abb. 1.

Abb. 1: $w_{1}$ aus BW.

'an|bin.den (V. 111) $1(500)$ jm d n. od. etwas mit Faden, Schnur usw. befestigen; binden Sie erst den Hund an!; Rosen am Spalier $\sim$; ein Bool an einen Pfahl $\sim$; ein Segel $\sim 1.1$ sich nicht $\sim$ lassen (rig.) seine Freiheis bewahren $2(800) \mathrm{m}$ it $\mathrm{jmd} \mathrm{m}$. (umg.) Streit beginnen. Händel suchen $3 \rightarrow$ a. ange. bunden

Die dreistelligen Zahlen "111", "500" und " 800 " in wa ${ }_{1}$ sind BW-spezifisch standardisierte Elemente der lexikographischen Beschreibungssprache dieses allgemeinen einsprachigen Wörterbuchs. Nur wer ein kundiger Benutzer des BW (i.S.v. Wiegand 1998: 506f.) ist, so daß er die Metatexte des BW gut kennt, weiß etwas mit diesen Zahlen anzufangen. Was er wissen muß, wird im folgenden theoriesprachlich ausgedrückt (also nicht so wie ein Benutzer über das Wissen verfügt bzw. es exteriorisieren kann). 
Ein Benutzer muß wissen, daß 2.B. "500" eine verdichtete Verweisangabe (v.VerwA) ist, die nur aus der elementaren Verweisadressenangabe (VerwAdA) besteht, weil die Verweisbeziehungsangabe (VerwBA), realisiert z.B. als " $\uparrow$ " oder "s." (= siehe), im Zuge der Textverdichtung weggelassen wurde. Der. "Benutzer muß weiterhin den Adressenfundort kennen, d.h.: er muß wissen, wo er die mit der Angabe "500" erwähnte Verweisadresse innerhalb des Wörterbuches suchen muß, nämlich im Vorspann des BW, und zwar als Element einer numerischen äußeren Zugriffsstruktur zu einer Tabelle, in der die Satzmuster für Verben aufgelistet sind. Nach Ausführung einer Verweisbefolgungshandlung (i.S.v. Wiegand 1998: 413) kann er die Verweisadresse "500" (auf S. 26) erreichen und unter dieser Umtextadresse das Verweisziel, nämlich folgende Satzmusterangabe finden: "S + Vb + Akko” (= Subjekt + finites Verb + Akkusativobjekt). Weiterhin muß der Benutzer aber auch wissen, daß er die Satzmusterangabe auf das Lemmazeichen von $\mathrm{wa}_{1}$, also auf anbinden beziehen muß; denn es handelt sich ja um eine Angabe, mit der auch ein Satzmuster für anbinden gegeben wird, und gerade dieser Sachverhalt war ja der Zweck der Verweisung. Woher aber kann der Benutzer wissen, daß er den genannten Bezug herstellen muß? Er kann dies nur dann wissen, wenn er wei $\beta$, daß die verdichtete Verweisangabe " 500 " in wa ${ }_{1}$ auch an die Lemmazeichengestaltangabe (LZGA) adressiert ist. Damit haben wir - sozusagen en passant - ein erstes Ergebnis zur Adressierung von Verweisangaben; es gilt nämlich: Jede artikelinterne Verweisangabe ist erstens explizit an mindestens eine Verweisadresse adressiert, die in der Verweisadressenangabe (als einer Teilangabe der Verweisangabe) erwähnt ist, und zweitens an eine andere artikelinterne Angabe, anhand derer (oder: mit deren Hilfe) die lexikographische Information, die anhand des Verweisziels ermittelt werden kann, vom Benutzer auf den Adressaten zu beziehen ist. Verweisangaben sind also stets mindestens zweifach adressiert. Nur dadurch können sie ihre Funktion erfüllen, dem Benutzer als Brücke zwischen zwei Textsegmenten zu dienen, die aufeinander zu beziehen sind, so da $B$ eine lexikographische Information entstehen kann.

$\mathrm{Da}$ auf die lexikographische Textverdichtung unter 2.2 einzugehen ist, kann nachfolgend gleich auf einige Aspekte der Standardisierung des Gegenstands von Wörterbuchartikeln eingegangen werden.

$\mathrm{Zu}$ (c): Nach Wiegand (1998: 302, D 3-5) ist der Wörterbuchgegenstand eines bestimmten Wörterbuchs die Menge der in diesem Wörterbuch lexikographisch bearbeiteten Eigenschaftsausprägungen von einer oder mehreren sprachlichen Eigenschaften bei einer bestimmten Menge von im Wörterbuch erwähnten sprachlichen Ausdrücken, die zu einem bestimmten Wörterbuchgegenstandsbereich gehören. Bei einem Aussprachewörterbuch der neuhochdeutschen Standardsprache ist die sprachliche Eigenschaft die Aussprache und der Wörterbuchgegenstandsbereich die neuhochdeutsche Standardsprache. Der Wörterbuchgegenstand ist dann die Ausprägung dieser Eigenschaft ,Aussprache' bei jedem einzelnen neuhochdeutschen Lemmazeichen, also die jewei- 
lige Lemmazeichenaussprache. Wird der Wörterbuchgegenstand eines Wörterbuchs (wie z.B. bei Aussprache- und Rechtschreibwörterbüchem) gerade durch eine Eigenschaft festgelegt, liegt ein monoinformatives Wörterbuch vor. Geschieht die Festlegung des Wörterbuchgegenstands durch mehrere sprachliche Eigenschaften (wie z.B. bei allgemeinen einsprachigen Wörterbüchern) liegt ein polyinformatives Wörterbuch vor. Mit der Festlegung der Eigenschaften, die zu bearbeiten sind, ist noch keine Standardisierung gegeben. Vielmehr ist diese erst dann etabliert, wenn relativ zu Lemmazeichentypen genau festgelegt ist, welche der Angabeklassen stets zu berücksichtigen sind und welche nicht berücksichtigt werden dürfen. Es ist klar, daß eine strikte Standardisierung bezüglich des Wörterbuchgegenstandes nicht bei allen Wörterbuchtypen erwünscht ist.

$Z u$ (d): Die Standardisierung der textuellen Strukturen und Architekturen eines Wörterbuches ist durch die Standardisierung des Wörterbuchgegenstandes in bestimmter Weise präformiert. Denn durch die Festlegung der Angabeklassen pro Lemmazeichentyp ist zugleich festgelegt, welche Angabeklassen und wieviele zur Trägermenge einer abstrakten hierarchischen Mikrostruktur eines Artikeltyps gehören. Definiert man auf solchen Trägermengen die erforderlichen strukturprägenden Relationen (vgl. 2.3), dann legt man damit die artikelinterne Datendistribution fest, so daß eine textstrukturelle Standardisierung gegeben ist, die sich auf die Mikrostruktur als Teilstruktur der Artikelkonstituentenstruktur bezieht. Auch letztere Struktur kann standardisiert werden; entsprechende Standardisierungsinstruktionen legen dann zusätzlich fest, welche nichttypographischen Mikrostrukturanzeiger (z.B. Kommata, senkrechte Striche; runde/eckige/spitze Klammern u.a.) wie zu setzen sind.

Die Standardisierung der Mikrostruktur determiniert die Standardișierung der Adressierungsstruktur (wie wir noch genauer sehen werden) und damit auch die der Angabestruktur weitgehend.

Die artikelspezifische Standardisierung bezieht sich meistens auch auf die Präsentation der lexikographischen Daten als zweidimensionale Textgestalten und Text-Teilgestalten im zweidimensionalen Druckraum, d.h. standardisiert sind z.B. die artikelinterne Absatzbildung, die Textblockbildung, die Auslagerung bestimmter Angaben auf den Außensteg der Wörterbuchseite u.v.a.m. Anders ausgedrückt heißt das, daß die Mikroarchitekturen (vgl. zu diesen z.B. Wiegand 2000: 259ff.) in ihren verschiedenen Ausprägungen standardisiert sind.

Der Grad der Standardisierung in Wörterbuchartikeln kann unterschiedlich sein. Auch kann in einem Wörterbuch vorgesehen sein, daß bestimmte Bereiche stark, andere weniger stark standardisiert sind. Beispielsweise sind die Formkommentare ( $\mathrm{fk}$ ) im BW stark standardisiert, wie die folgenden vier Beispiele zeigen: 
$\mathrm{fk}_{\mathrm{l}}$ : 'Bei-stand <m.; -(e)s, $\left.\ddot{-} \mathrm{e}\right\rangle$

$\mathrm{fk}_{2}$ : 'Wein.brand <m.; -(e)s; $\ddot{-} \mathrm{e}>$

$\mathrm{fk}_{3}$ : 'Spiel.ball <m.; -(e)s; $\because \mathrm{e}>$

fks: 'Stief.sohn <m.; -(e)s; $\ddot{-e}$.

Die Befolgung der Standardisierungsinstruktionen hat bei den Formkommentaren $\mathrm{fk}_{1}$ bis $\mathrm{fk}_{4}$ dazu geführt, daß alle linguistischen Aussagen zu den Lemmazeichen, die zur gleichen Aussageklasse gehören (außer den Aussagen zur Rechtschreibung), so lexikographisch vertextet wurden, daß die vier Formkommentare stark standardisiert sind und damit insgesamt untereinander weitestgehend ähnlich, weil sie in folgenden Hinsichten gleich sind:

- Die Anzahl der Angaben (aus derselben Klasse von Angaben mit gleichem allgemeinem genuinem Zweck) ist gleich.

- Die Reihenfolge dieser Angaben ist gleich.

- Die Zuordnung der typographischen Mikrostrukturanzeiger (halbfett und normal) zur Angabeform der elementaren Angaben ist gleich.

- Die nichttypographischen Mikrostrukturanzeiger (spitze Klammer, Semikolon und Komma) sind gleichwertig verwendet und damit nach Position und Funktion gleich.

- Die positionsgebundenen Angabesymbole (freistehender Akzent, Mittelpunkt, Bindestrich und hochgestellter Zweierpunkt) sind gleichartig verwendet und damit nach Position und Funktion gleich.

- Die Anwendung der Kondensierungsmethoden (i.S.v. Wiegand 1996c) ,Einschachteln' (vgl. „-(e)s”), ,Abkürzen' (vgl. „m.”) und ,Ersetzen' (vgl. "-(e)s" und "-e") hat zu gleichen Textsegmenten geführt.

- Entsprechendes kann für die Auslassungsmethode relativ zu Volltexten gezeigt werden (aus der linguistischen Aussage "Beistand ist maskulin" wird zunächst „Beistand $m$. "; vgl. 2.2).

- In $\mathrm{fk}_{1}$ bis $\mathrm{fk}_{4}$ sind die Morphologieangaben bei Substantiven (MorA.S) also die nichtelementare Angabe in " $\langle>$ " - sogar hinsichtlich ihrer Angabeform und ihrer Angabefunktion (und damit als ganze Angabe) vollständig gleich.

\subsection{Zur Textverdichtung}

Im folgenden geht es nur um die innere Textverdichtung (i.S.v. Wiegand 1998a), also um die, welche sich auf die lexikographischen Teiltexte mit einem Leitelementträger und damit auf Wörterbuchartikel bezieht; weiterhin wird nur die Textkondensierung, nicht die Textkomprimierung betrachtet. Denn nur die erste Art der Textverdichtung, in der das Verhältmis von Schriftzeichen zu propositionalen Gehalten so modifiziert wird, daß die propositionale Dichte größer wird, ist für das Verständnis der Adressierung von Interesse. Jeder stark stan- 
dardisierte Wörterbuchartikel, der auch hinsichtlich der Textkondensation standardisiert ist, so daß ein in einem bestimmten Grad verdichteter (z.B. hochverdichteter) und stark standardisierter Wörterbuchartikel gegeben ist, kann als ein zweiteiliges textuelles Kondensat (bestehend aus einem vorderen und hinteren Teilkondensat; vgl. Abb. 2) aufgefaßt werden; welches durch die Anwendung von Textverdichtungsmethoden auf einen Volltext, wie Kürzen, Abkürzen, Auslagern, Ersetzen, Zusammenrücken, Zusammenfassen und Ineinanderschachteln, und unter Berücksichtigung des Codes für die typographischen und nichttypographischen Mikrostrukturanzeiger entstanden ist. Der Volltext zu einem hochverdichteten und stark standardisierten Wörterbuchartikel (wie z.B. $w a_{1}$ bis $w a_{5}$ ) besteht aus einem Titel und dem zugehörigen Kotext.

Bei der Anwendung von Textverdichtungsmethoden auf den Volltext von $w_{a_{2}}$ wird der Titel des Volltextes zum vorderen Teilkondensat und der Kotext zum hinteren Teilkondensat. Durch Anwendung des Codes für die typographischen und nichttypographischen Mikrostrukturanzeiger wird sodann das vordere Teilkondensat zum Lemma, und das hintere Teilkondensat wird zu einer geordneten Menge von funktionalen Textsegmenten, nämlich Angaben und nichttypographischen Mikrostrukturanzeigern.

Dies ist in Abb. 2 anhand des Einzeilenartikels $w_{2}$ aus dem HWDG veranschaulicht; zusätzlich findet sich in Abb. 2 ein erweitert kommentierter Strukturgraph zu den beiden Artikelkonstituentenstrukturen (der abstrakten und der isomorphen konkreten) von $w_{2}$, auf den unter 2.3 kurz zurückzukommen ist.

Das Konzept der Volltexte ist ein notwendiges Konstrukt der Theorie der Wörterbuchform, das eine angemessene Erklärung der lexikographischen Textkondensierung ermöglicht. Wenn davon gesprochen wird, Wörterbuchartikel seien verdichtet (oder: kondensiert), dann ist eine geeignete Interpretation dieser Redeweise, daß ein Textverarbeitungsproze $B$ vorausgesetzt wird, in dem ein Text ${ }_{i} z u$ einem Text $T_{j}$ (unter Anwendung von Textverdichtungsmethoden) verdichtet wurde; $T_{i}$ ist dann einer von mehreren möglichen Volltexten, die ausgehend von einem Wörterbuchartikel konstruiert werden können. Wird die Volltextbildung standardisiert, so daß nur ein Volltexttyp gebildet werden darf (was hier nicht vorgeführt werden kann; vgl. dazu Wiegand 1998a: 15ff.), werden die Wörterbuchartikel hinsichtlich der Textverdichtung vergleichbar, und der Textverdichtungsgrad wird berechenbar. Ein möglicher Volltext $\mathbf{z u ~ w a}_{2}$ ist $\mathrm{Vt}_{1}$.

$\mathrm{Vt}_{1}:$ [1] Wörterbuchartikel zu Brigg.

[2] Die Rechtschreibung von Brigg ist |Brigg |.

[3] Der Artikel zu Brigg ist die.

[4] Die Form des Ng. Sg. ist Brigg.

[5] Die Form des G. Sg. ist Brigg.

[6] Die Form des Nom. Pl. ist Briggs.

[7] Brigg bedeutet soviel wie Segelschiff mit zwei Masten. 
Abb. 2: Veranschaulichung zur Textverdichtung als Übergang von einem Volltext zu einem hochverdichteten Wörterbuchartikel als zweiteiligem Kondensat

\section{A.H. EINFACHE INTEGRERTE ARTIKELKONSTITUENTENSTRUKTUR}
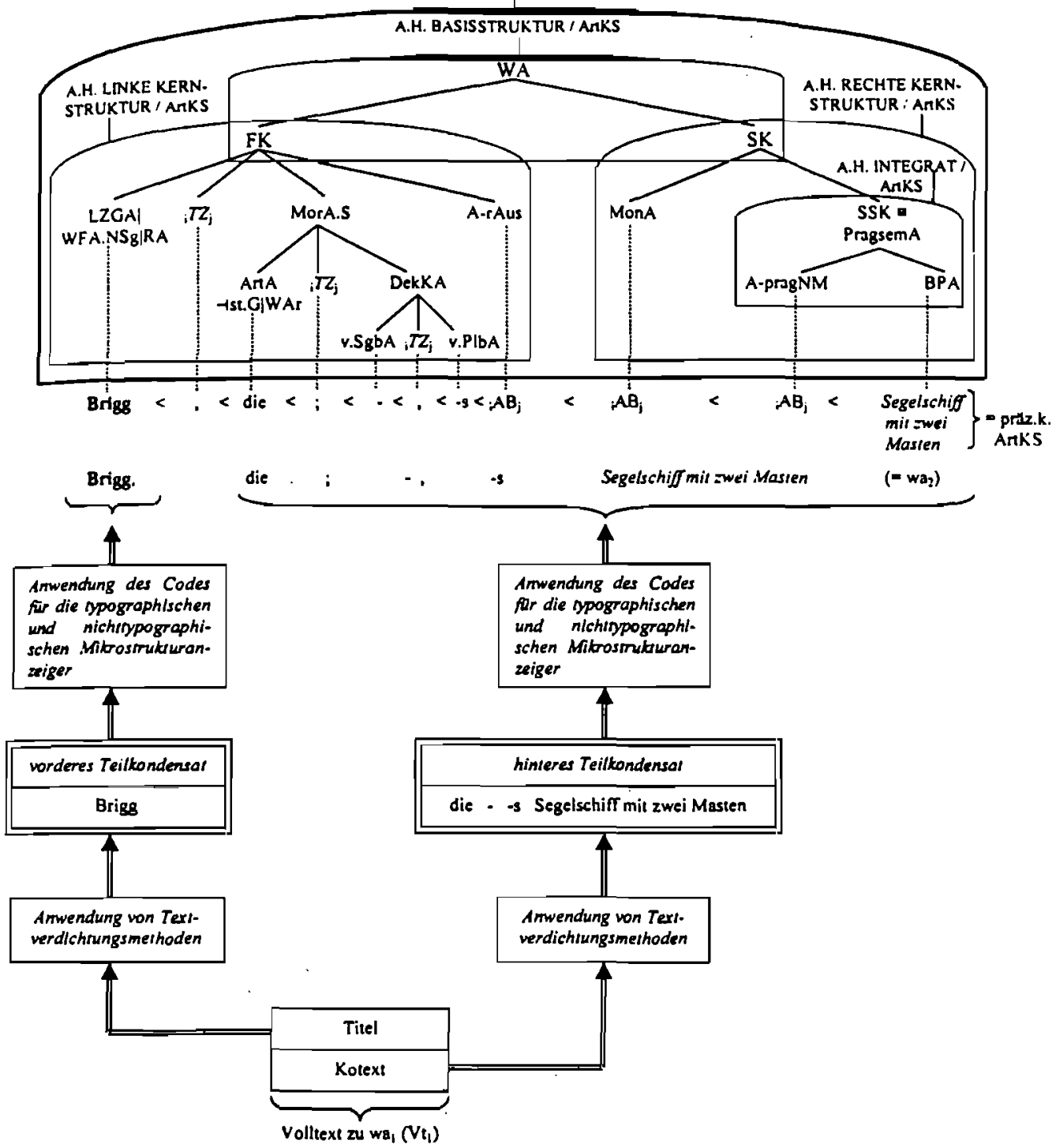

Erläuterungen: „, $x-Y$ “ bedeutet (von unten nach oben gelesen) soviel wie $x$ ist ein Teil von $Y_{;}, X^{\prime \cdots . . .} Y^{\prime \prime}$ bedeutet (von unten nach oben gelesen) soviel wie $x$ ist ein Element von $Y(=\mathrm{x} \in$ $Y$ ); , $\mathrm{x} \equiv \mathrm{y}^{\text {" }}$ bedeutet soviel wie $\mathrm{x}$ ist (hier) identisch mit $y_{;}, \ldots \mathrm{x}<\mathrm{y}^{\prime \prime}$ bedeutet soviel wie $x$ geht $y$ voraus; ...

Abkürsungen: ArtKS = Artikelkonstinentenstruktur; präz. = präzedentiv; $k .=$ konkret; WA = Wörterbuchartikel; FK = Fornkommentar; SK = semantischer Kommentar; SSK = semanti- 
scher Subkommentar; LZGA = Lemmazeichengestaltangabe; ,|“ bedeutet soviel wie zugleich; WFA. $N S_{B}=$ Wortformenangabe für den Nom. Sg.; $R A=$ Rechtschreibangabe; ${ }_{i} T Z_{j}=$ Trennzeichen mit den Vorgänger- und Nachfolgervariablen „;“" und ,j"; MorA.S = Morphologieangabe bei Substantiven; ArtA = Artikelangabe; „-l" bedeutet soviel wie anhand derer er. schließbar ist; st.G = stabiles Genus; WAr = Wortart; DekKA = Deklinationsklassenangabe; v.SgbA = verdichtete Singularbildungsangabe; v.PIbA = verdichtete Pluralbildungsangabe; $\mathrm{A}$. rAus = Angabe zur regelmäBigen Aussprache; MonA = Monosemieangabe; Pragsem $A=$ pragmatisch-semantische Angabe; A-pragNM = Angabe zur pragmatischen Nullmarkierung; $\mathrm{BPA}=$ Bedeutungsparaphrasenangabe; ${ }_{\mathrm{A}} \mathrm{AB} \mathrm{B}_{\mathrm{j}}=$ Angabeblank mit den Vorgänger- und Nachfolgervariablen „i" und ,j"; A. = ABSTRAKT; H. = HIERARCHISCH.

In $\mathrm{Vt}_{1}$ ist [1] der Titel, und [2] bis [7] bilden den Kotext des Volltextes zu wa ${ }_{1}$. Werden auf $\mathrm{Vt}_{1}$ die geeigneten Textverdichtungsmethoden Satz für Satz angewendet, entsteht $w_{2}$. Bei dem Textverdichtungsprozeß geht die syntaktische Struktur von $\mathrm{Vt}_{1}$ weitgehend verloren und damit auch die syntaktischen Funktionen und die kohäsiven Mittel. Damit gehen auch die Kohäsionsanweisungen verloren, die es dem Benutzer ermöglichen, die mikrostrukturellen Konstituenten des Wörterbuchartikels aufeinander zu beziehen. Für den Verlust der textuellen Voraussetzungen, um die artikelinternen Angaben aufeinander beziehen zu können, sind die artikelinternen Adressierungsbeziehungen - wie wir noch genauer sehen werden - der für das Verständnis eines Wörterbuchartikels notwendige Ersatz.

\subsection{Zu den Mikrostrukturen}

Hochverdichtete und stark standardisierte Wörterbuchartikel weisen zahlreiche textuelle Strukturen auf. ${ }^{3}$ Im folgenden kann nur soweit auf die Mikrostukturen eingegangen werden, wie es für das Verständnis der artikelinternen Adressierung erforderlich ist.

Alle konkreten Textkonstituentenstrukturen von Wörterbuchartikeln (Artikelkonstituentenstrukturen, Mikrostrukturen, Suchbereichsstrukturen) sind hierarchische Strukturen und dadurch erhältlich, daß Wörterbuchartikel durch die Anwendung einer der Varianten der funktional-positionalen Segmentationsmethode (i.S.v. Wiegand 1990: 20ff.) in funktionale Textsegmente zerlegt werden, so daß auf bestimmten genau festgelegten Mengen solcher Textsegmente strukturprägende Relationen definiert werden können. Die wichtigste Voraussetzung dafür besteht darin, daß man über ein geeignetes Inventar von Klassen von funktionalen Textsegmenten mit gleichem allgemeinen genuinen $Z$ weck verfügt.

Mikrostrukturen sind Ordnungsstrukturen, so daß bestimmte Strukturausschnitte für den kundigen Benutzer als innere Zugriffs- oder Schnellzugriffsstrukturen fungieren können. Mikrostrukturen sind weiterhin Teilstrukturen von Artikelkonstituentenstrukturen. In Abb. 2 sind Strukturen des letzteren Typs, die $\mathrm{zu} \mathrm{wa}_{2}$ gehören, unter Zuhilfenahme formaler Darstellungsmittel 
abgebildet; es handelt sich um die abstrakte hierarchische einfache integrierte Artikelkonstituentenstruktur und um die isomorphe konkrete Struktur des gleichen Typs, weiterhin um einige hierarchische Teilstrukturen dieser beiden Strukturen sowie schließlich um die konkrete präzedentive Artikelkonstituentenstruktur als Teilstruktur der konkreten hierarchischen Artikelkonstituentenstruktur. Die konkrete hierarchische Artikelkonstituentenstruktur eines bestimmten Wörterbuchartikels ist diejenige Textkonstituentenstruktur, die erstens die artikelinterne Distribution der Angaben festlegt und zweitens diese Distribution mittels der nichtypographischen Mikrostrukturanzeiger explizit kenntlich macht. Letzteres ist deswegen der Fall, weil zur Trägermenge einer konkreten hierarchischen Artikelkonstituentenstruktur auch die nichttypographischen Mikrostrukturanzeiger gehören. Im Falle von $\mathrm{wa}_{2}$ gehören also das fette Komma nach dem Lemma, das Semikolon nach der Artikelangabe und das Komma als Teil der Deklinationsklassenangabe zur Trägermenge (vgl. Abb. 2). Im Unterschied zur Artikelkonstituentenstruktur legt die konkrete hierarchische Mikrostruktur nur die artikelinteme Distribution der Angaben fest.

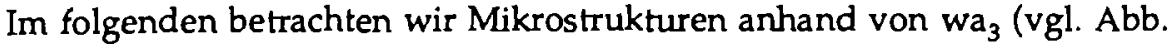
3) etwas genauer:

\section{Abb. 3: $w_{3}$ aus HWDG}

Burg, die; -, - 1 1. /rïhgeschichtlicher od. miltelalterlicher Bau bes. der Feudalherren, der vorwiegend als Anlage zur Verteidigung diente: alte, perfallono Burgen; dio B. wurde belagert, erstürzat - 9. Jagerapr. Bau des Bibers

Ausgehend von einem Inventar von Klassen von Angaben mit gleichem allgemeinen genuinen Zweck segmentieren wir $\mathrm{wa}_{3}$ unter Anwendung der Methode der nichtexhaustiven funktional-positionalen Segmentation (i.S.v. Wiegand 1990: 22). Das Segmentationsergebnis findet sich in dem kommentierten Baumgraphen in Abb. 4; die Kommentierung besteht darin, daß jeder durch die Segmentation entstehenden elementaren und nichtelementaren Angabe in runden Klammem ein Kleinbuchstabe als Individuenname zugeordnet wird. Das verwendete Inventar von Angabeklassen und damit auch die Segmentation ist sprachtheoretisch motiviert (vgl. Wiegand 2000: 236f.). Sprachliche Zeichen - wie im vorliegenden Fall Burg - haben eine Form und eine Bedeutung. In $\mathrm{wa}_{3}$ werden mit der Angabe a zunächst explizite lexikographische Aussagen zur Form von Burg gemacht; daran schließen sich Aussagen an, die sich vor allem auf die Bedeutung von Burg beziehen. Daher wird die erste Segmentationsfuge zwischen $a$ und $b$ gelegt; $a$ und $b$ sind dann unmittelbare Textkonstituenten von $w_{3}$. Alle unmittelbaren Textkonstituenten eines ganzen Wörterbuchartikeltextes heißen (aus Gründen, die hier nicht relevant sind) Kommentar, wenn sie nicht nur aus einem nichttypographischen Struk- 
turanzeiger bestehen; $a$ ist der Formkommentar (FK) von $w_{3}, b$ der semantische Kommentar (SK). Weist ein Wörterbuchartikel nur einen Form- und einen semantischen Kommentar auf (wie z.B. wa $_{3}$ ), heißen die zugehörigen Mikrostrukturen einfach. Den einfachen stehen die erweiterten Mikrostrukturen gegenüber. Beispielsweise hat $w_{5}$ (vgl. Abb. 13) einen Postkommentar und weist damit eine rechtserweiterte Mikrostruktur auf (vgl. Wiegand 1989b). Die Abkürzungen $F K$ und $S K$ gelten als Klassensymbole, so daß gilt: $a \in F K$ und $b \in S K$. Beide Kommentare haben den Status von nichtelementaren Angaben, d.h.: sie sind weiter funktional-positional segmentierbar, ohne daß nichtfunktionale Textreste entstehen. In Abb. 4 ist das Ergebnis der funktional-positionalen Segmentation von $\mathrm{wa}_{3}$ insgesamt dargestellt. Lediglich der nächste Segmentationsschritt beim Formkommmentar wird hier noch genauer erläutert. ${ }^{4}$ Im nächsten Segmentationsschritt wird a $\in$ FK in drei Angaben segmentiert, so $\mathrm{da} B$ im Strukturgraphen in $\mathrm{Abb} .4$ drei Kanten vom a-Knoten wegführen, und

Abb. 4: Kommentierter Strukturgraph zur konkreten hierarchischen einfachen integrierten Mikrostruktur von $\mathrm{wa}_{3}$

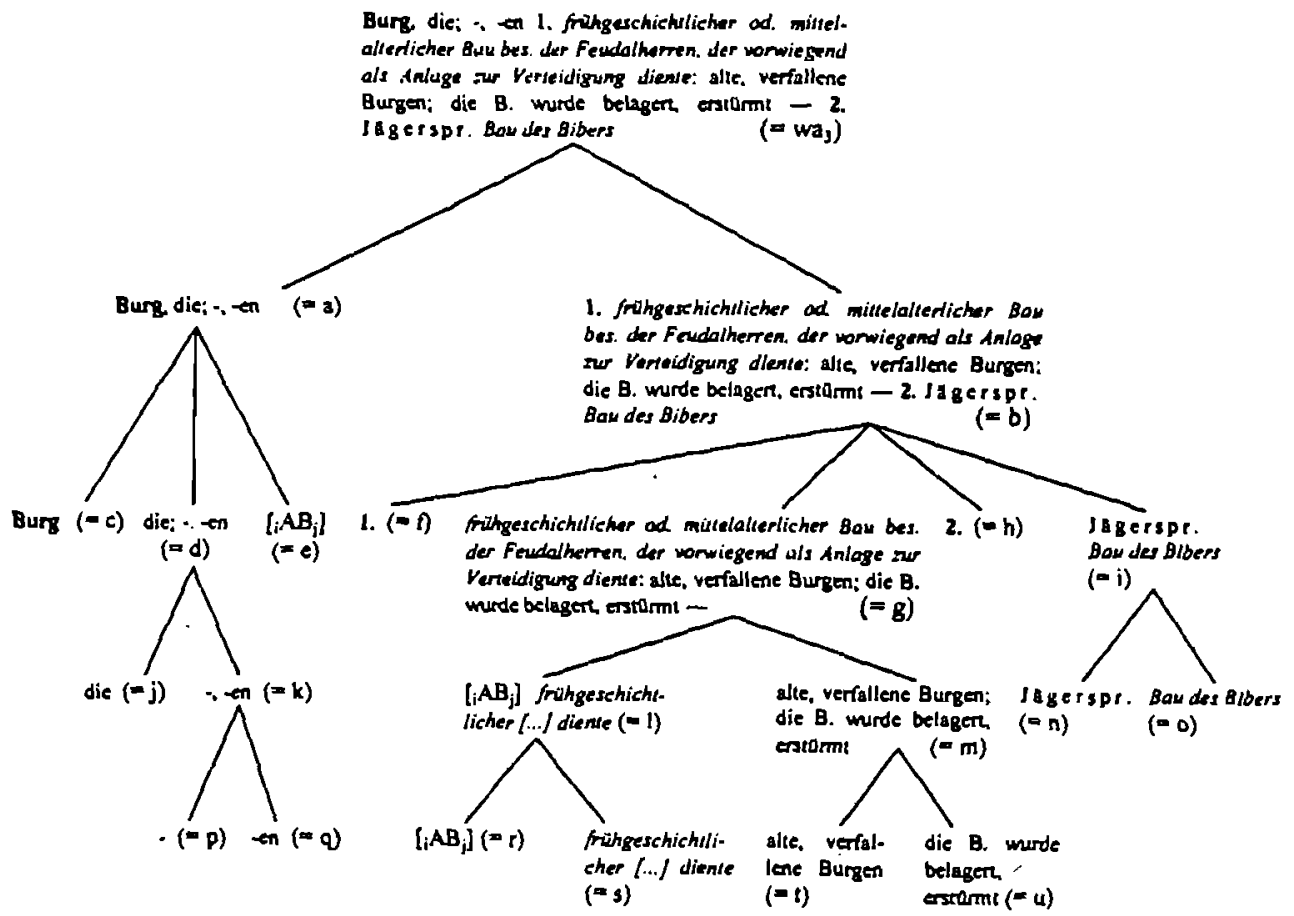

Erläuterungen: Die Kleinbuchstaben „a“ bis „u“ in den runden Klammem sind Individuennamen für (nichtelementare und elementare) Angaben aus wa ${ }_{3}$, und "wa ${ }^{\prime}$ "ist der Individuenname fur den HWDG-Artikel zu Burg, so daß „= $x^{\prime \prime}$ hier $z u$ lesen ist wie hat als Namen $x$. 
zwar zu c, d und e; c vereinigt alle lexikographischen Aussagen zum Formativ des Lemmazeichens Burg. Anhand der Lemmazeichengestaltangabe (LZGA) Burg (die im vorliegenden Fall die gleiche Form wie das Lemma aufweist) erfährt der Benutzer die Rechtschreibung und - gemäß eingespielter Gepflogenheiten in Printwörterbüchern des Neuhochdeutschen für die Präsentation von substantivischen Lemmazeichen - die Wortform im Nominativ Singular. Bei $d$ handelt es sich um die Morphologieangabe bei Substantiven (MorA.S), so $\mathrm{da} B$ gilt $d \in$ MorA.S. Auf $d$ folgt $e$, und zwar als Angabeblank ( $A B$ ) indiziert mit einer "Vorgänger-Variablen „ii" und einer Nachfolger-Variablen „j” $\left({ }_{i} A B_{j}\right)$. Ein Angabeblank wird bei der Darstellung einer konkreten Mikrostruktur stets dann in einer bestimmten textuellen Artikelposition angesetzt, wenn aus dem Fehlen einer Angabe einer bestimmten Angabeklasse in dieser Position für den kundigen Benutzer ein Schluß auf eine spezifische Eigenschaft des Lemmazeichens möglich ist. Bei HWDG-Artikeln ist dies deswegen möglich, weil im Metatext (S. XXV) steht: „Ausspracheangaben werden für alle Wörter verzeichnet, die von Ausspracheregeln der deutschen Sprache abweichen." Gemäß dieser Feststellung lautet der Formkommentar des HWDG-Artikels zu Cape wie folgt: $\mathrm{k}_{5}$ : Cape, das; $-\mathrm{s}$, $-\mathrm{s}$ [ke:p].

In der Position der Ausspracheangabe (ke:p $\in$ AusA) wird in der Strukturdarstellung zu wa ${ }_{3}$ ein Angabeblank angesetzt, der zu folgenden Angabeklassen gehört: Klasse der Angaben zur regelmäßigen Aussprache (A-rAus). Es gilt daher: v.PlbA $A_{\mathrm{PA}} \in \mathrm{A}-\mathrm{rA}$ us.

In einer Theorie der Wörterbuchform kann es nicht darum gehen, daß nur die konkreten Strukturen je spezifischer Wörterbuchartikel betrachtet werden. Um zu theoretisch fundierten Generalisierungen übergehen zu können, benötigt man abstrakte Kategorien. Im Bereich der Mikrostrukturen sind diese dadurch erhältlich, daß man mit Angabeklassen arbeitet. Dies bedeutet, daß man Trägermengen für abstrakte Mikrostrukturen bildet, deren Elemente $\mathrm{n}$ Angabeklassen (AK) sind (mit $n \geq 1$ ) und $m$ Artikelklassen (mit $m=1$ ).

Dadurch, daß wir die in Abb. 4 eingeführten Individuennamen ( „a" bis " $u$ " und " $\mathrm{wa}_{3}{ }^{\prime \prime}$ ) verwenden, können wir nachfolgend $\mathrm{wa}_{3}$ einer Artikelklasse und seine Angaben den entsprechenden Klassen von Angaben mit gleichem allgemeinen genuinen Zweck zuordnen, so daß folgende 22 Element-KlassenBeziehungen gelten:

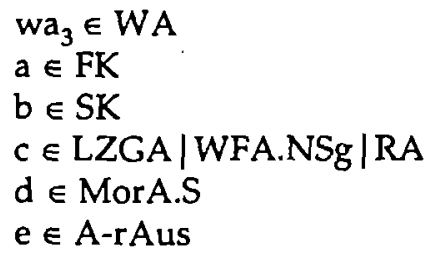

$$
\begin{aligned}
& f \in P A \\
& g \in S S K \\
& h \in P A \\
& i \in S S K \\
& j \in \text { ArtA } \mid \text { st.G } \mid \text { WAr } \\
& k \in \text { DekKA }
\end{aligned}
$$




$l \in$ PragsemA
$m \in$ KBeiA ${ }^{2}$
$n \in v . F G A$
$o \in B P A$
$p \in v . S g b A$

$1 \in \operatorname{Pragsem} A$

Damit verfügen wir über die Trägermenge $\left(\mathrm{M}_{\mathrm{AK}}\left(w \mathrm{a}_{3}\right)\right)$ der abstrakten (a) hierarchischen (h) (einfachen integrierten) Mikrostruktur (MiS), die $w_{3}$ aufweist $\left(\mathrm{MiS}_{h}^{\mathrm{a}}\left(\mathrm{wa}_{3}\right)\right)$. Diese Trägermenge kann demnach wie folgt (in aufzählender Schreibweise) angegeben werden, wobei jede Angabeklasse nur einmal erscheint:

$\mathrm{M}_{\mathrm{AK}}\left(w_{a_{3}}\right)$ für $\mathrm{MiS}_{\mathrm{h}}^{\mathrm{a}}\left(\mathrm{wa}_{3}\right)=$ (WA, FK, SK, LZGA|WFA.NSg|RA, MorA.S, A-rAus, PA, SSK, ArtA - st.G|WAr, DekKA, PragsemA, KBeiA2, v.FGA, v.BPA, v.SgbA, v.PlbA, A-pragNM, BPA, KBeiA, v.KBei²A\}.

Auf der Menge $\mathrm{M}_{\mathrm{AK}}\left(w \mathrm{wa}_{3}\right)$ definieren wir nun zwei disjunkte strukturprägende Relationen, und zwar erstens eine zweistellige partitive Relation (Teil-GanzesRelation) und zweitens eine zweistellige Präzedenzrelation (VorgängerNachfolger-Relation). Die erstgenannte Relation ist reflexiv, antisymmetrisch und transitiv; ihre Elemente sind 2-Tupel von Angabeklassen, z.B. $\langle F K, W A\rangle$, $<$ MorA.S, FK >, <PragsemA, SSK>. Auf ein Paar von je einem Element aus den beiden verschiedenen Klassen eines 2-Tupels trifft der Relationsterm " $\mathrm{x}$ ist eine Teilangabe von $\mathrm{y}^{\prime \prime} \mathrm{zu}$; beispielsweise ist: „d $\in$ MorA.S ist eine Teilangabe $z \mathrm{u}$ a $\epsilon \mathrm{FK}^{\prime \prime}$ eine wahre Aussage. Die zweitgenannte Relation, die Präzedenzrelation, ist irreflexiv, asymmetrisch und transitiv; ihre Elemente sind 2-Tupel von

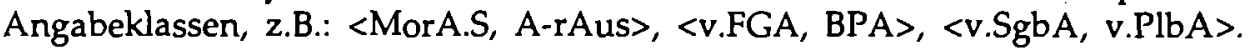
Auf ein Paar von einem Element aus den beiden verschiedenen Klassen eines 2-Tupels trifft der Relationsterm "x geht voraus $y$ " zu (nachfolgend symbolisiert als " $\left.x<y^{\prime \prime}\right)$; beispielsweise gilt: $(p \in v . S g b A)<(q \in v$.PlbA).

Die abstrakte hierarchische Mikrostruktur, die zur konkreten hierarchischen Mikrostruktur von $w_{3}$ isomorph ist, läßt sich dann - wie in Abb. 5 mittels eines Baumgraphen darstellen, für den die üblichen Darstellungskonventionen gelten, so daß (um der Übersichtlichkeit willen) bei der Darstellung der partitiven Relation der transitive Mantel nicht explizit mittels Kanten repräsentiert wird (z.B. fehlen die erschließbaren Kanten: „v.PlbA - FK" oder v.FGA - WA), und eine eigene graphische Repräsentation der Präzedenzrelation unterbleibt (vgl. auch Wiegand 1989a). Vielmehr gelten die Konventionen des Schriftsystems sinngemäß, so daß das, was durch die Etikettierung eines am weitesten links liegenden Tochterknotens bezeichnet wird, dem vorausgeht, was durch die weiteren weiter rechts liegenden Tochterknoten des gleichen Vaterknotens bezeichnet wird.

Strukturgraphen für abstrakte textuelle Strukturen von Wörterbuchartikeln (wie z.B. den in Abb. 5) können auf zwei Arten kommentiert werden: Sie können mit einfachen oder erweiterten Kommentierungen versehen werden. 
Einfache Kommentierungen bestehen aus Umrandungszeichen, die etikettiert sind; umrandet werden die jeweils dargestellte textuelle Struktur und einige oder alle ihre Teilstrukturen; die Etikettierungen bestehen aus den Strukturnamen und/oder Abkürzungen für diese. Erweiterte Kommentierungen sind erläuternde Zusätze zu einfach kommentierten Strukturgraphen. Weder die einfache noch die erweiterte Kommentierung tangieren die formalen Eigenschaften der Strukturgraphen.

Abb. 5: Nichtkommentierter Strukturgraph zur abstrakten hierarchischen einfachen integrierten Mikrostruktur, die $\mathrm{wa}_{3}$ aufweist

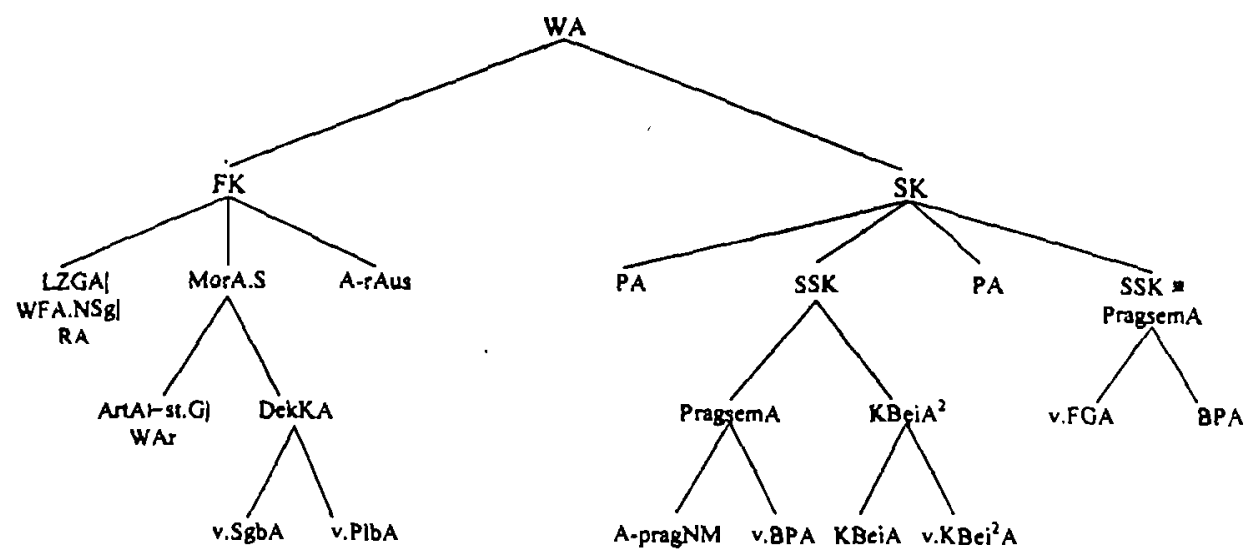

Abkürzungen: v.FGA $=$ verdichtete Fachgebietsangabe; $K B e i A^{2}=$ Kompetenzbeispielangabe, die aus zwei Kompetenzbeispielangaben besteht; v.KBei ${ }^{2} \mathrm{~A}=$ verdichtete Kompetenzbeispielangabe, anhand derer zwei Kompetenzbeispiele erschlossen werden können (oder: mit zwei Kompetenzbeispielen).

$\mathrm{Zu}$ einer abstrakten hierarchischen Textkonstituentenstruktur können stets mehrere konkrete hierarchische Textkonstituentenstrukturen verschiedener Wörterbuchartikel isomorph sein (vgl. z.B. Wiegand 1991: 459); dies bedeutet u.a.: Aussagen über Struktureigenschaften der abstrakten Struktur gelten auch für die isomorphen konkreten Strukturen. Der Isomorphismus und auf der Darstellungsebene die formalen Eigenschaften von Baumgraphen bilden die Voraussetzung dafür, daß eine abstrakte hierarchische zusammen mit der isomorphen konkreten hierarchischen Textkonstituentenstruktur eines Artikels in einem Strukturgraphen, der zwei Baumgraphen kombiniert, dargestellt werden können. Bei einer solchen Strukturdarstellung, die in der Theorie der Wörterbuchform am häufigsten verwendet wird, werden von der konkreten Textkonstituentenstruktur nur die terminalen Textkonstituenten explizit berücksichtigt; die nichtterminalen können aufgrund des Isomorphismus erschlossen werden. In Abb. 6 sind die beiden Strukturdarstellungen aus Abb. 4 und Abb. 5 $\mathrm{zu}$ einer Strukturdarstellung vereinigt und mit einer erweiterten Kommentie rung versehen. 
Abb. 6: Erweitert kommentierter Strukturgraph zur (abstrakten und zur isomorphen konkreten) hierarchischen einfachen integrierten Mikrostruktur, die $\mathrm{wa}_{2}$ aufweist

\section{A.H. EINFACHE INTEGRIERTE MIKROSTRUKTUR}

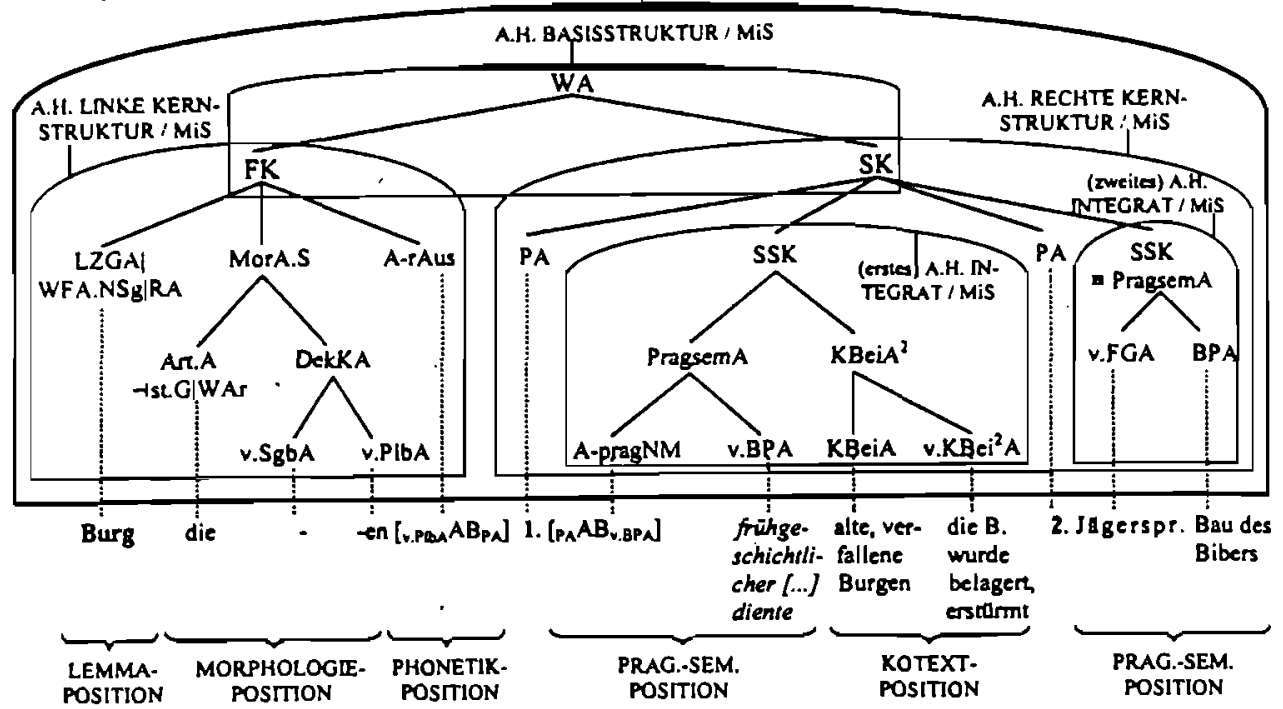

Erläuterungen: , $x-y^{\prime \prime}$ bedeutet (von unten nach oben gelesen) soviel wie $x$ ist ein Teil von $y$; "x $\cdots y^{\prime \prime}$ bedeutet (von unten nach oben gelesen) soviel wie $x$ ist ein Element von $(=x \in y)$; ${ }_{, x} \mathrm{x} \equiv y^{\prime \prime}$ bedeutet soviel wie $x$ ist (hier) identisch mit $y ;,{ }^{\prime \prime} \mathrm{X} / \mathrm{Y}$ " ist zu lesen wie $X$ als Teilstruktur von $Y$.

Abkürungen: A. = ABSTRAKT; H. = HIERARCHISCH; WA = Worterbuchartikel; $\mathrm{FK}=$ Formkommentar; SK = semantischer Kommentar; MiS = Mikrostruktur, LZGA = Lemmazeichengestaltangabe; WFA.NSg $=$ Wortformenangabe: Nominativ Singular; RA $=$ Rechtschreibangabe; MorA.S = Morphologieangabe bei Substantiven; A-rAus = Angabe zur regelmaBigen Aussprache; ArtA -st.G|WAr = Artikelangabe, anhand derer das stabile (st.) Genus (G) sowie die Wortart (WAr) erschlieBbar sind; , DekKA = Deklinationsklassenangabe; v.SgbA = verdichtete Singulartildungsangabe; v.PlbA = verdichtete Pluralbildungsangabe; SSK = semantischer Subkommentar; Pragsem A = pragmatisch-semantische Angabe; A-pragNM = Angabe zur pragmatischen Nullmarkierung; v.BPA = verdichtete Bedeutungsparaphrasenangabe; $\mathrm{KBeiA}^{2}=$ (nichtelementare) Kompetenzbeispielangabe, die aus zwei Kompetenzbeispielangaben besteht; $\mathrm{KBeiA}=\mathrm{Kompetenzbeispielangabe;} \mathrm{v} \cdot \mathrm{KBei}^{2} \mathrm{~A}=$ ver dichtete Kompetenzbeispielangabe, anhand derer zwei Kompetenzbeispiele erschließbar sind (oder: mit zwei Kompetenzbeispielen); v.FGA = verdichtete Fachgebietsangabe; $A B=$ Angabeblank; v.PlbA $A B_{P A}=$ Angabeblank, dessen Vorgănger eine v.PlbA und dessen Nachfolger eine $\mathrm{PA}$ ist; $\mathrm{PAAB}_{\mathrm{V} . \mathrm{BPA}}=$ Angabeblank, dessen Vorgänger eine PA und dessen Nachfolger eine v.BPA ist. 
$\mathrm{Zu}$ jeder Strukturdarstellung von abstrakten Textkonstituentenstrukturen kann ein allgemeines Strukturbild und ein erweitertes allgemeines Strukturbild angegeben werden, die nach festgelegten Darstellungskonventionen erstellt werden (vgl. z.B. Wiegand 2000). Das allgemeine Mikrostrukturbild für $w_{3}$ findet sich in Abb. 7. Die Strukturbilder dienen vor allem zur schnellen Orientierung über die Mikrostrukturart.

Abb. 7: Allgemeines Mikrostrukturbild für einfach integrierte Mikrostrukturen von Wörterbuchartikeln zu zweifach polysemen Lemmazeichen wie z.B. wa $_{3}$

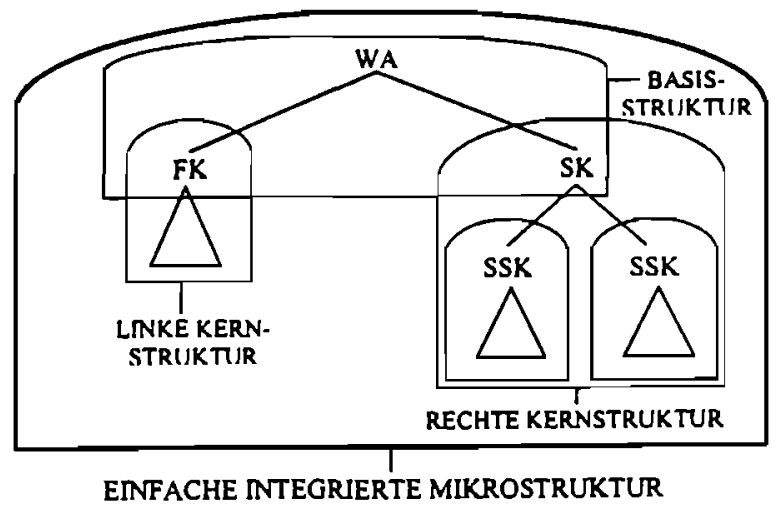

Die bisher über Standardisierung, Textverdichtung und Mikrostrukturen von Wörterbuchartikeln eingeführten Kenntrisse sind zwar noch relativ eingeschränkt, dürften aber ausreichen, um die sich anschließende Darstellung der artikelinternen Adressierung verstehen zu können.

\section{Adressierung in der einsprachigen Lexikographie}

Wir betrachten nachfolgend vor allem die artikelinterne Adressierung, und

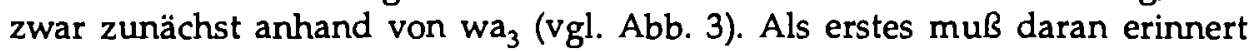
werden, daB der Ausdruck Adresse nicht nur - wie auch adressieren - in der Alltagssprache unterschiedlich verwendet wird (vgl. Wiegand 1989a: 445), sondern auch in verschiedenen theoretischen Zusammenhängen unterschiedlich terminologisiert ist. In der Dokumentationswissenschaft und in der Datenbanktheorie wird unter einer Adresse von (oder: für) Daten, die sich auf einem Speichermedium befinden, die Angabe des Speicherplatzes verstanden, die es ermöglicht, gezielt auf die Daten zuzugreifen. Zwar kann man auch in lexikographischen Zusammenhängen davon sprechen, daß ein Lemma, wie z.B. Burg in $w_{3}$, eine Adresse darstellt, unter der ein Benutzer-in-actu etwas sucht und gegebenenfalls etwas findet; diese eher werkstattsprachliche (durchaus gut verständliche) Redeweise ist aber für eine präzise lexikographietheoretische Sprechweise weniger gut geeignet. Denn ein Lemma in einem Sprachwörter- 
buch kann nicht nur als eine Adresse im gerade genannten Sinn gelten, weil ein Benutzer anhand des Lemmas selbst auch Informationen über die Lemmazeichengestalt, z.B. über eine bestimmte Wortform oder über die Form eines Affixes sowie über die Rechtschreibung erschließen kann. Während also anhand eines Lemmas Informationen über den Wörterbuchgegenstand erschließbar sind, gilt das z.B. für eine Adresse einer Datenbank nicht notwendigerweise.

Wenn man von Adressen im Zusammenhang mit der artikelinternem Adressierung spricht, sind Adressen immer Angaben. In wa ${ }_{3}$ sind alle Angaben (außer dem Formkommentar) an die Lemmazeichengestaltangabe adressiert (vgl. Abb. 15). Die zweistellige Beziehung " $x$ ist adressiert an $y$ " (mit " $x$ " und " $y$ " als Variablen für Angaben) heißt Adressierungsbeziehung. Beispielsweise gelten für $\mathrm{wa}_{3}$ folgende Aussagen:

- MorA.S ist adressiert an die LZGA.

- DekKA ist adressiert an die LZGA.

- PragsemA ist adressiert an die LZGA.

Der Sachverhalt der Adressierung kann auch konvers mit "y ist die Adresse von $x$ " wiedergegeben werden (mit " $y$ " als Variable für Adressen und " $x$ " als Variable für Angaben). Entsprechend gilt dann z.B.: Die LZGA ist die Adresse der MorA.S.

In der lexikographischen Werkstattsprache nennt man eine Adresse zusammen mit einer an sie adressierte Angabe auch eine lexikographische Bearbeitungseinheit. In $w^{2} a_{3}$ ist z.B. <Burg, Bau des Bibers> eine lexikographische Bearbeitungseinheit (vgl. auch 3). Es ist hierbei allerdings unbedingt zu beachten, daß die Konverse der Adressierungsrelation nicht mit der Bearbeitungsrelation verwechselt wird. Denn bearbeitet werden Phänomene des Wörterbuchgegenstandes und richt Angaben, während Angaben an Angaben adressiert sind und nicht an Phänomene des Wörterbuchgegenstandes; daher muß auch die Angabebeziehung " $x$ ist Angabe $z u y^{\prime}$ (auf die hier aus Platzgründen nicht näher eingegangen werden kann) strikt von der Adressierungsbeziehung unterschieden werden (vgl. Wiegand 2000e).

Die beiden Elemente einer lexikographischen Bearbeitungseinheit sind als verschiedene Angaben lexikographische Daten und keine lexikographischen Informationen. Wie bereits erwähnt, ist eine lexikographische Information eine kognitive Entität, die während der Ausführung von Benutzungshandlungen beim Benutzer-in-actu entsteht. Sieht man von Informationen über geschriebene Wortformen ab, die sich aus der jeweiligen Angabeform als solcher beim Lesen unmittelbar ergeben, dann sind lexikographische Informationen nur dadurch erhältlich, daß ein Benutzer-in-actu eine Angabe auf dasjenige bezieht, wozu sie eine Angabe ist (also die Angabebeziehung rekonstruiert). Die Herstellung dieses Bezugs wird durch die Kenntnis der Adressierungsbeziehung 
möglich und kann nur so erfolgen, daß eine Angabe $a_{1}$ auf eine andere Angabe $a_{2}$ bezogen wird, weil $a_{2}$ die Adresse von $a_{1}$ ist. Erst danach kann der zweite Schritt der Informationsgewinnung erfolgen; dieser besteht in der kognitiven Konstruktion des Adressaten als dem wichtigsten Teil der Antwort auf eine Suchfrage.

Will beispielsweise ein Benutzer-in-actu die verdichtete Singularbildungsangabe "-" in wa $_{3}$ (also: - $\epsilon$ v.SgbA) für eine Antwort auf seine Suchfrage "Wie lauten die Singularformen von Burg?" nutzen, dann muß er die verdichtete Singularbildungsangabe auf die Lemmazeichengestaltangabe, also auf Burg beziehen. Unter der Voraussetzung, daß der Benutzer-in-actu weiß, daß ,"- die Singularbildungsangabe ist, ergibt sich erst nach Ausführung der genannten kognitiven Operation eine lexikographische Information. Diese besteht in dem punktuellen Wissen, daß die vier Singularformen von Burg, die der Benutzerin-actu bilden muß, Burg lauten (vgl. Wiegand 2000e).

In Wörterbuchartikeln einsprachiger Wörterbücher (wie z.B. wa $a_{1}$ bis $w_{5}$ ) ist die zentrale Adresse meistens die Lemmazeichengestaltangabe (LZGA). Diese weist in mehreren Hinsichten einen Sonderstatus auf. Sie ist erstens Träger des Leitelementes, also der Buchstabenfolge, nach der alphabetisiert wird (vgl. Wiegand 1989). In wa ${ }_{3}$ beispielsweise ist die LZGA Burg hinsichtlich der Eigenschaften, die bei der Alphabetisierung eine Rolle spielen können, nämlich hinsichtlich der Anzahl und hinsichtlich der Reihenfolge der Buchstaben, mit dem Leitelement |Burg| gleich. Dies ist natürlich nicht notwendigerweise so, sondern nur dann, wenn die Methode der exhaustiven mechanischen Alphabetisierung (wie in den meisten modernen Wörterbüchern) angewandt wurde (vgl. dazu Wiegand 1989). Das Leitelement ist stets ein Element der Trägermenge der Makrostruktur und damit auch stets ein Element der (oder: einer der) äußeren Zugriffsstruktur(en). Die Einheit, die das Leitelement trägt und damit ein Zugriffselement ist, und anhand derer man zugleich etwas über die schriftkonstituierte Wortform des Lemmazeichens erfährt, heißt Lemma. Spricht man von der Lemmazeichengestaltangabe, dann wird von dem Zugriffsaspekt abstrahiert, d.h.: die Eigenschaft, ein Leitelementträger zu sein, spielt dann keine Rolle, sondern nur die Eigenschaft, daß die Gestalt des Lemmazeichens präsentiert wird. Zum Sonderstatus der Lemmazeichengestaltangabe gehört zweitens, daß sie neben dem Formkommentar, dessen Teilangabe sie ist, die einzige Angabe unter allen Wörterbuchangaben ist, die nicht eine andere Angabe als Adresse hat. Die Lemmazeichengestaltangabe ist adressenlos, ist aber dennoch eine Angabe. 5

Angaben, welche die gleiche artikelinterne Adresse haben, können verschiedene Adressaten haben. So gilt beispielsweise für die in Abb. 8 berücksichtigten Angaben aus $w a_{3}$ folgendes: Die Artikelangabe, die verdichtete Singularbildungsangabe, die verdichtete Pluralbildungsangabe und die verdichtete Bedeutungsparaphrasenangabe sind an die Lemmazeichengestaltangabe adressiert; ihre Adressaten sind jedoch 2.T. verschieden. Denn es gilt: 
- Der Adressat der Artikelangabe ist das Lemmazeichenparadigma (alle acht Wortformen von Burg sind femininum).

- Der Adressat der verdichteten Singularbildungsangabe ist die Form des Genitiv Singulars.

- Der Adressat der verdichteten Pluralbildungsangabe ist die Form des Nominativ Plurals.

- Der Adressat der verdichteten Bedeutungsparaphrasenangabe ist das Lemmazeichenparadigma (allen acht Wortformen von Burg kommt die Bedeutung zu, die anhand der v.BPA erschließbar ist).

Abb. 8: Veranschaulichung zum Zusammenhang von Angaben, lemmatischer Adresse und Adressaten anhand von $\mathrm{wa}_{3}$

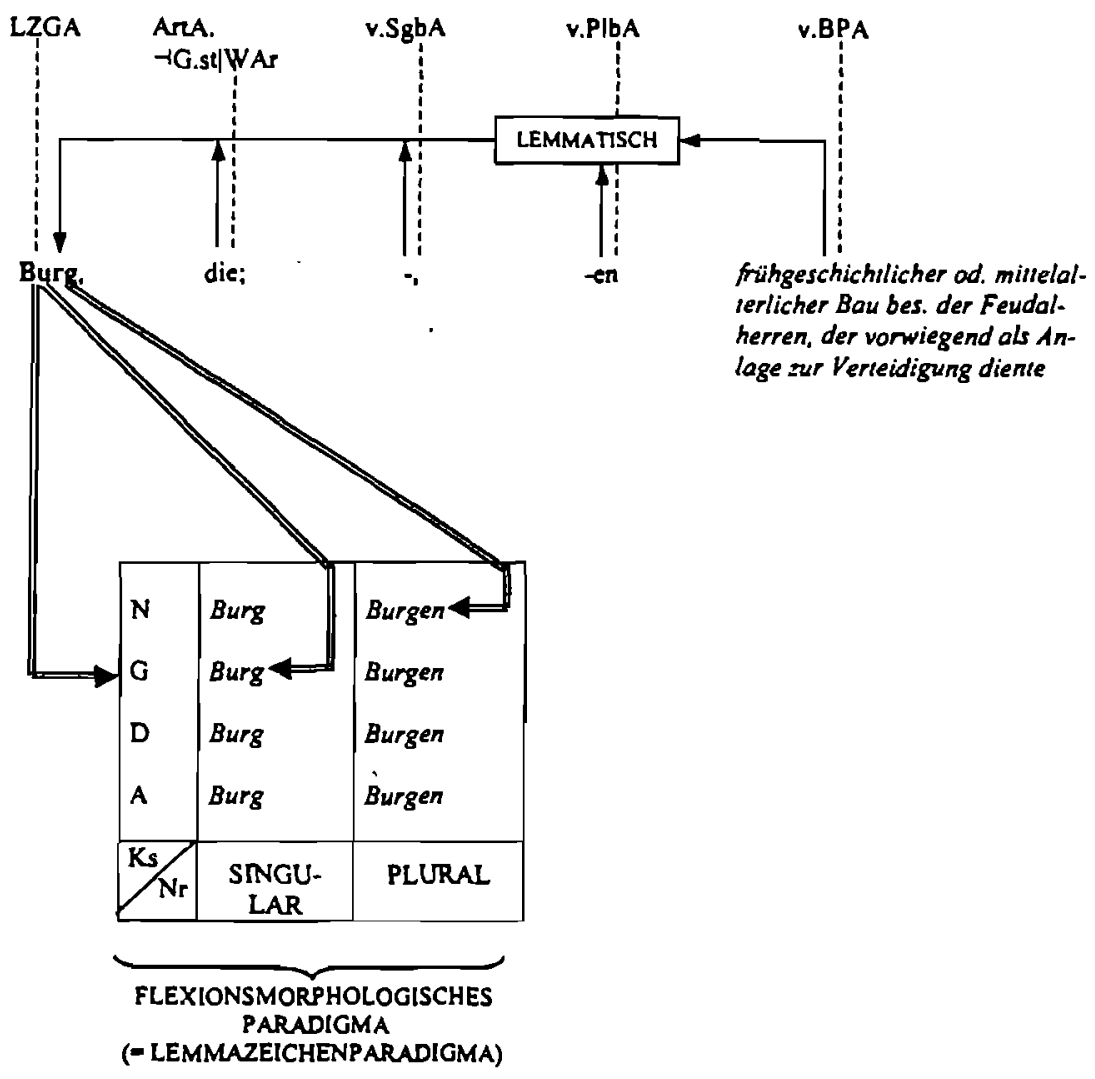

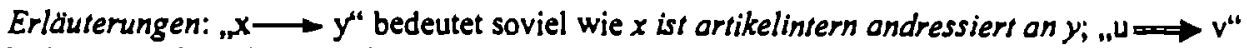
bedeutet soviel wie $u$ ist die Adresse zum Adressaten $v_{i}, z-\ldots-Z^{\prime \prime}$ bedeutet soviel wie $z \in Z$ ( $z$ ist ein Element von $Z$ ); Abkürzungen: $\mathrm{N}=$ Nominativ; $\mathrm{G}=$ Genitiv; $\mathrm{D}=$ Dativ; $\mathrm{A}=$.Akkusativ; $\mathrm{Ks}=$ Kasus; $\mathrm{Nr}=$ Numerus.

Es lassen sich verschiedene Arten der artikelinternen Adressierung unterscheiden. Auf einige Arten wird nachfolgend kurz eingegangen. Die Unterscheidun- 
gen gelten nur für Wörterbücher, deren Wörterbuchgegenstandsbereich Sprachen oder Varietäten bilden, deren Schriftsystem eine zeilengebundene Verschriftung von links nach rechts vorsehen. Eine Angabe ist artikelintern linksadressiert, wenn ihre Adresse gegen die Schriftrichtung (oder: Schreibrichtung) und damit auf den Zeilen nach links orientiert gesucht werden muß. Entsprechend ist eine Angabe artikelintern rechtsadressiert, wenn ihre Adresse mit der Schriftrichtung und damit auf den Zeilen nach rechts orientiert gesucht werden muß. Die Rechtsadressierung ist relativ selten. Sie findet sich beispielsweise im Campe-WdS. Hier haben zahlreiche Artikel einen Präkommentar, der aus Angabesymbolen besteht, die nach rechts an die Lemmazeichengestaltangabe adressiert sind. Beispiele sind die folgenden Einträge (e):

$\mathrm{e}_{1}: \dagger$ Beschlagen; v. intrs. unregelm [...]

$\mathrm{e}_{2}$ : O Die Beisitzerstelle, Mz die $-n[\ldots]$

$\mathbf{e}_{3}: \Delta$ Durchschláfen, v. ntr. [...]

Auch die Artikelangabe "Die" in $e_{2}$ ist nach rechts an die Lemmazeichengestaltangabe „Beisitzerstelle” adressiert.

Die Unterscheidung von Links- und Rechtsadressierung gilt vor allem für die artikelinterne Adressierung. Artikelinterne Verweisangaben sind - wie wir gesehen haben - stets zweifach adressiert. Sie sind daher - wie alle anderen Angaben auch - zunächst einmal entweder artikelintern rechts- oder linksadressiert. Bezüglich ihrer Verweisadressen, die außerhalb des Artikels liegen, zu dem sie gehören, spricht man dagegen nicht von Links- oder Rechtsadressierung. Vielmehr wird in dem Fall, in welchem der Adressenfundort innerhalb der Lemmaliste des gleichen Wörterverzeichnisses liegt, zu dem der Artikel mit der fraglichen Verweisangabe gehört, entweder von Hinauf- oder Hinabadressierung gesprochen. Besonders in älteren Wörterbüchem benutzte man entsprechend als Verweisbeziehungsangabe Pfeile mit ikonischen Eigen-

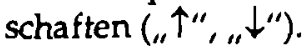

Bei der artikelinternen Adressierung kann weiterhin zwischen lemmatischer, sublemmatischer und nichtlemmatischer Adressierung unterschieden werden. Alle drei Arten können als Links- oder Rechtsadressierung ausgeprägt sein. Abb. 9 veranschaulicht die drei Möglichkeiten anhand der wesentlich öfter gegebenen Linksadressierung.

Lemmatische Adressierung liegt vor, wenn die artikelinterne Adresse die Lemmazeichengestaltangabe ist. Sublemmatische Adressierung ist gegeben, wenn die subartikelinterne Adresse die Zeichengestaltangabe zum Sublemmazeichen ist; und schließlich handelt es sich um nichtlemmatische Adressierung, wenn die Adresse eine artikelinterne Angabe ist, die nicht als Leitelementträger fungiert. Sind alle Angaben eines Wörterbuchartikels (außer der Lemmazeichengestaltangabe und dem Formkommentar, zu dem die LZGA gehört) an die Lemmazeichengestaltangabe adressiert, liegt vollständige lemmatische Adressierung vor. 
Abb. 9: Veranschaulichung zu den Arten der artikelinternen Linksadressierung bei hochverdichteten und stark standardisierten Wörterbuchartikeln: Lemmatische, nichtlemmatische und sublemmatische Linksadressierung

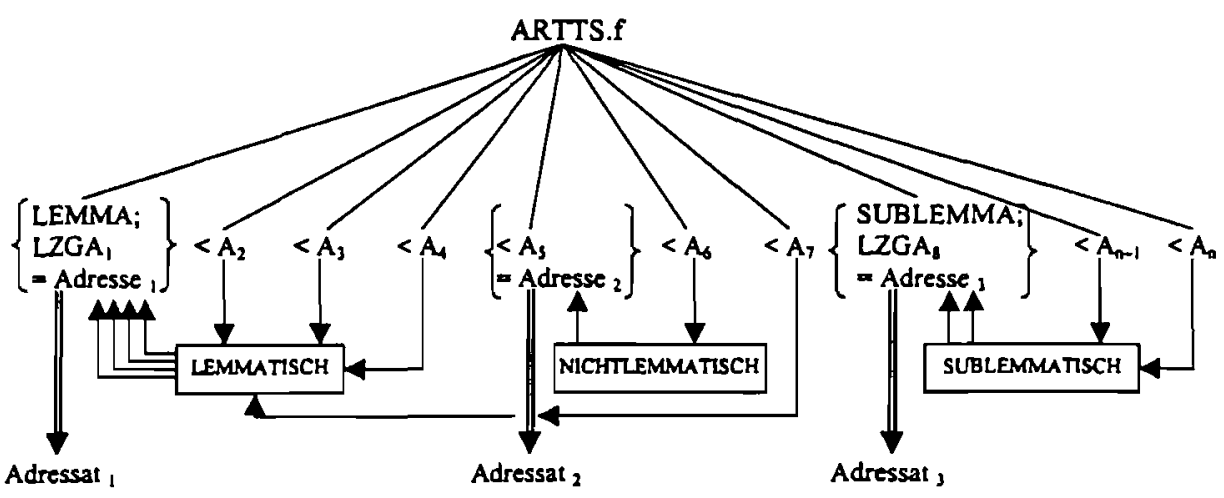

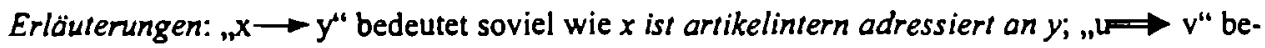
deutet soviel wie $u$ ist die Adresse zum Adressaten $v_{;}$"<" bedeutet soviel wie geht voraus; Abkürzungen: $\mathrm{A}=$ Angabe; ARTTS.f $=$ funktionale Artikelteilstrecke; $\mathrm{LZGA}=$ Lemmazeichengestaltangabe.

Dies heißt zugleich, daß es keinen artikelinternen Themenwechsel gibt: Alle lexikographischen Aussagen, die in der verdichteten Form von lexikographischen Bearbeitungseinheiten gegeben sind, sind daher vom Benutzer-in-actu auf das Lemmazeichen und das Lemmazeichenparadigma zu beziehen. Für Subartikel gilt dies sinngemäß. Hier spricht man entsprechend von vollständiger sublemmatischer Adressierung.

Mit den bisherigen Unterscheidungen verfügen wir über erste Ansätze zu einer Typologie der artikelinternen Adressierungsbeziehungen, die in den Abb. 10 und 11 - zusammen mit einer weiteren noch zu erläuternden Differenzierung - in eine Übersicht gebracht sind.

Die Standardisierung, wie sie unter 2.1 erläutert wurde, betrifft auch die Adressierungsbeziehungen (vgl. Abb. 11). Daher kann zwischen standardisierter und nichtstandardisierter Adressierung unterschieden werden. Eine Adressierungsbeziehung gilt als standardisiert, wenn nach dem Mikrostrukturenprogramm des Wörterbuchs die Klasse der Adressen für eine bestimmte Angabeklasse vorgeschrieben ist; dies ist z.B. dann der Fall, wenn es eine Standardisierungsinstruktion gibt, die besagt, daß alle Bedeutungsparaphrasenangaben im semantischen Kommentar an die Lemmazeichengestaltangabe zu adressieren sind. Adressen, die im Mikrostrukturenprogramm als Adressen für Angaben aus bestimmten Klassen festgelegt sind, sind standardisierte Adressen. Nichtstandardisierte Adressen sind dagegen solche, die vom bearbeitenden Lexikographen frei gewählt werden können (wobei es hier unterschiedliche Freiheitsgrade geben kann); für die zugehörigen Angaben, die an die nichtstandardisierten Adressen adressiert sind, gilt dies entsprechend. Die frei wählbaren 
Abb. 10: Erster Ausschnitt aus einer Typologie der artikelinternen Adressierungsbeziehungen

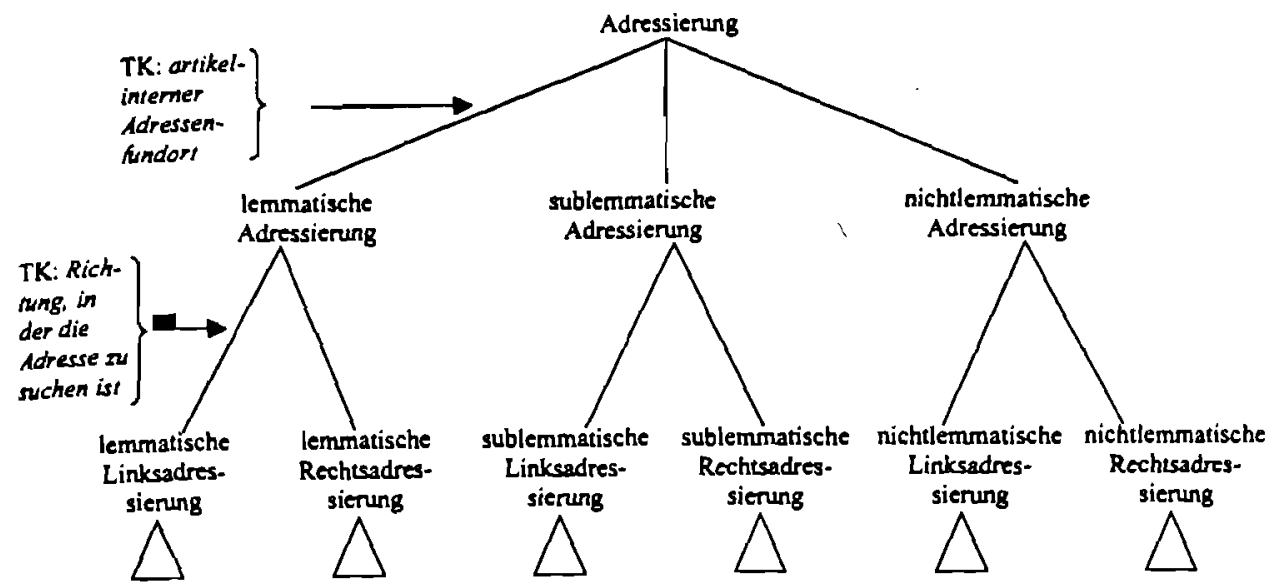

Erläuterungen: „ $\longrightarrow$ “bedeutet soviel wie die Anwendung des TK führt zu der Unterteilung, Abkürzungen: $\mathrm{TK}=$ Typologiekriterium.

Abb. 11: Zweiter Ausschnitt aus einer Typologie der artikelinternen Adressierungsbeziehungen

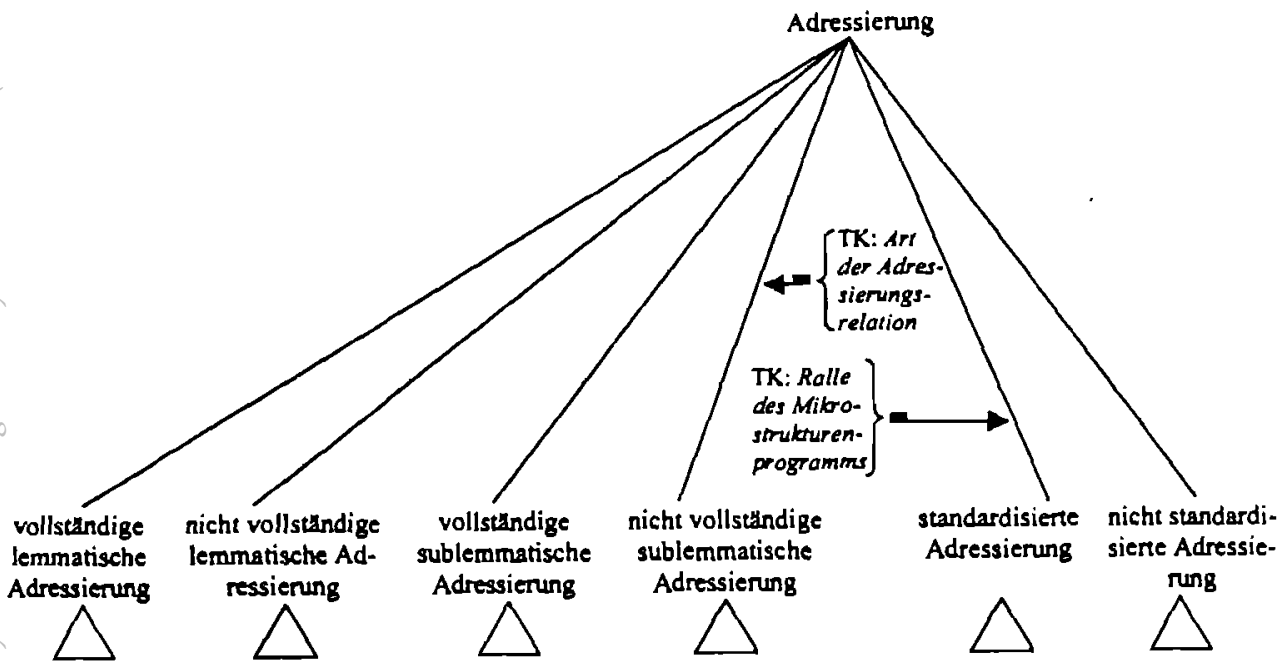

Erläuterungen: $\longrightarrow$ “ bedeutet das gleiche wie , $\longrightarrow$ “(vgl. Abb. 10).

Angaben heißen Glossate (vgl. Wiegand 1989a: 447); die nichtstandardisierten Adressen heißen entsprechend Glossat-adressen. Je nach ihrer Position zu der Angabe, die die Glossatadresse darstellt oder von der ein Teil eine Glossat- 
adresse ist, können Prä-, Post- und Binnenglossate unterschieden werden; Präglossate sind rechts-, Binnen- und Postglossate dagegen linksadressiert. Glossate kommen in vielen Wörterbüchem häufig vor. Zahlreiche Glossate finden sich z.B. in $w_{4}$ in Abb. 12.

Abb. 12: $\mathrm{Wa}_{4}$ aus dem HWDG

sauber /Adj./ 1. frei von Schmulz, Verunreinigungen: saubere Finger(nägel), Kleider, Schuhe, Straßen; sauberes Wasser; ein sauberes Besteck; Hemd; etw. s. bulrsten, putzen, waschen; sich s. halten; das Kind ist schon s. (braucht keine Windeln mehr zu tragen) 2.1. sorgfältig und ordentlich (ausgeführt): eine saubere Arbeit, Naht, Schrift; stw. s. aussägen, stopfen 2.2. im Klang rein (1.2): dieser Ton war nicht ganz s. 2.3. eine saubere (angemessene und überzeugende, widerspruchsfreie) Lösung des Problems 2.4. Inur $a d v$. $\mathrm{umg}$. das hast du s. (sehr gut) hingekriegt! 3.1. moralisch einwandfrei: eine saubere Gesinnung, Haltung; ein sauberer Charakter; iron. du hast ja saubere (anfechtbare) Ansichten!; dein sauberer Freund (dein Freund, dieser gemeine Kerl) hat uns das eingebrockt! 3.2. Inicht adv./ ein sauberes (von Schuld unbelastetes) Gewissen - 4. umg. süddt. ósterr. schweiz. hübsch (I.I), schmuck: ein sauberes Mädchen, ein sauberer Bursche; $\mathbf{s}$. aussehen $+(k)$ eine saubere $\lambda$ Weste haben

Für $w_{4}$ gelten folgende Aussagen:

- "braucht keine Windeln mehr zu tragen" ist ein (linksadressiertes) Postglossat zur verdichteten Kompetenzbeispielangabe "das Kind ist schon s." als Glossatadresse.

- „angemessene und überzeugende, widerspruchsfreie” ist ein nichtelementares (linksadressiertes) Binnenglossat zur Glossatadresse "saubere", das aus zwei elementaren ( $=$ nicht weiter segmentierbaren) Binnenglossaten besteht.

- "sehr gut" ist ein (linksadressiertes) Binnenglossat zur Glossatadresse „s.”. - "anfechtbare” ist ein (linksadressiertes) Binnenglossat mit der Glossatadresse "saubere".

- "dein Freund, dieser gemeine Kerl" ist ein (linksadressiertes) Binnenglossat mit der Glossatadresse "sauberer Freund".

- "von Schuld unbelastetes" ist ein (linksadressiertes) Binnenglossat mit der Glossatadresse "sauberes”. 
Mit den sechs Glossaten in $\mathrm{wa}_{4}$ sind sechs nichtstandardisierte Themenwechsel verbunden. Diese müssen von den standardisierten Themenwechseln unterschieden werden wie sie $z$.B. in $w a_{5}$ vorliegen. ${ }^{6}$

Abb. 13: $w_{5}$ aus dem HWDG

Streich, der; -(e)s, -e aus Übermut, Mutwillen begangene Handlung, durch die jmd. geneckt, hereingelegt. geärgert werden soll: ein lustiger, ubermütiger, böser $\mathrm{S}$.; freche, tolle Streiche machen, vollfuhren, veruben; er ist stets zu Streichen aufgelegt

+ jmd. spielt jmdm. einen S. (legt jmdn. herein); etw. spielt jmdm. einen S. etw. läßt jmdn. im Stich: sein Gedächtnis hat ihm einen $S$. gespielt

Im Postkommentar von $w_{5}$, der mit der Phrasemidentifizierungsangabe " + " beginnt, sind "legt jmdn. herein" und "etw. läßt jmdn. im Stich" nicht etwa verdichtete Glossate, sondern nach dem Mikrostrukturenprogramm des HWDG verdichtete Bedeutungsparaphrasenangaben für Phraseme, so daß in $w_{5}$ die verdichteten Phrasemangaben "jmd. spielt jmdm. einen S." und "etw. spielt jmdm. einen S." ihre standardisierten Adressen sind und somit in $\mathrm{wa}_{5}$ ein standardisierter Themenwechsel vorliegt.

Bei Wörterbuchartikeln mit vollständiger lemmatischer Adressierung und bei solchen mit vollständiger sublemmatischer Adressierung ist die zweistellige Adressierungsrelation nacheindeutig, so daß sie eine Funktion ist. Anhand von $w_{3}$ (vgl. Abb. 3) sei dies etwas eingehender erläutert. Die Adressierungsfunktion kann z.B. mittels eines Pfeildiagramms dargestellt werden (vgl. Abb. 14).

Nach der Analyse der Mikrostrukturen von $w a_{3}$ in 2.3. verfügen wir sowohl über die Trägermenge der abstrakten hierarchischen als auch über die der konkreten (k) hierarchischen (h) Mikrostruktur $\left(\mathrm{MiS}_{h}^{k}\right)$ von $w^{2} a_{3}$. Letztere Menge heiße $M_{A}\left(w a_{3}\right)$. Die 22 Elemente dieser Menge sind in 2.3. aufgezählt, und zwar unter Verwendung der in Abb. 4 eingeführten Individuennamen " $\mathrm{a}$ " bis " $u$ " und " $w a_{3}$ ". Wir vermindern nun $M_{A}\left(w a_{3}\right)$ um die Elemente $w a_{3} \in$ WA und $a \in F K$ und erhalten $M_{A}^{\prime}\left(w a_{3}\right)$. Auf dieser Menge $M^{\prime}{ }_{A}\left(w a_{3}\right)$ definieren wir nun die zweistellige irreflexive und asymmetrische Adressierungsrelation sie heiße $\mathrm{R}_{\mathrm{a}}\left(\mathrm{R}_{\mathrm{a}} \subseteq \mathrm{M}^{\prime}{ }_{\mathrm{A}}\left(w_{a_{3}}\right) \times \mathrm{M}_{\mathrm{A}}{ }_{\mathrm{A}}\left(\mathrm{wa}_{3}\right)\right)$, auf deren 2-Tupel der Relationsterm $\mathrm{T}_{\mathrm{a}}=x$ ist artikelintern standardisiert adressiert an $y$ zutrifft, wobei " $\mathrm{x}$ " eine Variable für Angaben (die keine Adressen sind) ist und " $y$ " eine Variable für Adressen. $\mathrm{Da} \mathrm{wa}_{3}$ ein Artikel mit vollständiger lemmatischer Adressierung ist, kann für "y" nur "LZGA |WFA.NSg|RA" eingesetzt werden; d.h. in jedem der 2-Tupel der Adressierungsrelation $R_{a}$ erscheint als zweite Koordinate LZGA | WFA.NSg $\mid$ RA, z.B. in dem 2-Tupel <MorA.S, LZGA $\mid$ WFA.NSg $\mid R A>$, auf das 
$T_{a}$ zutrifft. In der Abb. 14 entspricht diesem 2-Tupel die Darstellungskomponente "MorA.S $\rightarrow$ LZGA|WFA.NSg|RA". Die Definition von $R_{a}$ auf der Menge $M_{A}^{\prime}\left(w_{3}\right)$ führt zu deren Zerlegung in zwei disjunkte Teilmengen. Dies sind die beiden folgenden Mengen: $M_{A d}^{\prime}\left(w_{3}\right)$, die Menge der Angaben von $w a_{3}$, die Adressen sind (eine Einermenge), und $M_{-A d}^{\prime}\left(w a_{3}\right)$, die Menge der Angaben, die keine Adressen sind. Das Pfeildiagramm in Abb. 14 ist mithin eine formale Strukturdarstellung für die konkrete Adressierungsstruktur, die $\mathrm{wa}_{3}$ aufweist und damit ein Beispiel für die Darstellung der konkreten Adressierungsstruktur eines Wörterbuchartikels mit vollständiger lemmatischer Adressierung.

Abb. 14: Pfeildiagramm zur vollständigen lemmatischen Adressierung in $w_{3}$

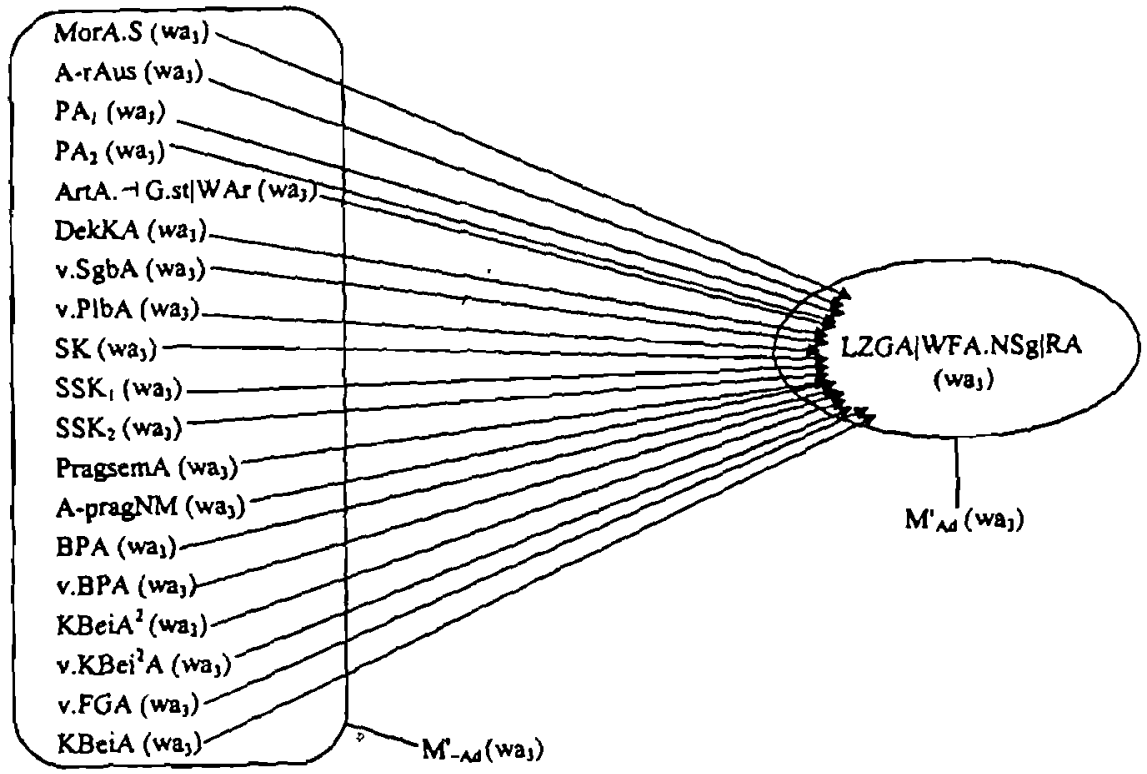

Erläuterungen: MorA.S ( $\left.w_{3}\right)$ ist zu lesen wie die fkonkrete) Morphologieangabe bei Substantiven in way; , $x \rightarrow y^{*}$ bedeutet soviel wie $x$ ist artikelintern standordisiert adressiert an $y$.

Abkürzungen: $M_{A d}^{\prime}\left(w_{3}\right)=$ Menge der Angaben von $w a_{3}$, die Adressen sind; $M_{-A d}^{\prime}\left(w a_{3}\right)=$ Menge der Angaben von way, die keine Adressen sind.

Es ist zu beachten, daB in $A b b .14$, um die konkreten Angaben besser identifizieren zu können, andere Individuennamen als in Abb. 4 für die konkreten Angaben in $w_{3} a_{3}$ verwendet sind, nämlich z.B. „DekKA $\left.\left(w_{3}\right)\right)^{\prime}$; dieser Name ist $z u$ lesen wie Deklinationsklassenangabe in $w a_{3}$ (nämlich $d \in$ DekKA). Bei einer Darstellung der abstrakten Adressierungsstruktur sind die Elemente der Träger- 
bei der Darstellung des semantischen Kommentars und der zugehörigen abstrakten hierarchischen rechten Kernstruktur als Teilstruktur der Angabe. struktur angewandt wurde. Das Klassensymbol für die Angabeklasse, zu der die Adresse gehört, wird (getrennt durch einen Mittelpunkt) unmittelbar hinter dem Klassensymbol für die adressierte Angabe aufgeführt; es wird also z.B. das erweiterte Knotenetikett „PragsemA-LZGA“ geschrieben und (vereinfacht) gelesen: "Pragmatisch-semantische Angabe, adressiert an die Lemmazeichengestaltangabe". Die Abkürzung für den Klassennamen einer Angabeklasse, die hinter dem Mittelpunkt steht, heißt Adressensymbol; im vorliegenden Fall ist das Adressensymbol „LZGA”. Anhand der Adressensymbole in einer Strukturdarstellung kann man unmittelbar ablesen, welche Adressierungsart in einem Wörterbuchartikel gegeben ist.

Für nichtelementare mikrostrukturelle Textkonstituenten, wie z.B. Formkommentare, semantische Kommentare, Morphologieangaben bei Substantiven usw. kann gelten, daß sie entweder adressenhomogen oder adressenheterogen sind. Eine nichtelementare mikrostrukturelle Textkonstituente ist adressenhomogen genau dann, wenn sie selbst und alle ihre Teilangaben an die gleiche Adresse adressiert sind; ist das nicht der Fall und kommen $n$ Adressen (mit $n \geq 2$ ) vor, ist die mikrostrukturelle Textkonstituente adressenheterogen. In $w a_{3}$ ist $z$.B. die Morphologieangabe bei Substantiven (MorA.S) und der semantische Kommen$\operatorname{tar}(\mathrm{SK})$ adressenhomogen. Der Formkommentar (FK) in $\mathrm{wa}_{3}$ ist dagegen adressenheterogen, da er selbst nicht, wie alle seine Teilangaben, an die Lemmazeichengestaltangabe adressiert ist. Um die Darstellung der hierarchischen Angabestrukturen möglichst übersichtlich gestalten zu können, kann daher von folgender Notationskonvention Gebrauch gemacht werden: Ist eine nichtelementare mikrostrukturelle Textkonstituente $T_{i}$ adressenhomogen, wird bei der Darstellung der abstrakten hierarchischen Mikrostruktur nur das Angabeklassensymbol für $T_{i}$ um das Adressensymbol erweitert. Dies besagt, daß alle Teilangaben von $T_{i}$ die gleiche Adresse haben wie $T_{i}$. In der Abb. 16 ist von dieser Notationskonvention Gebrauch gemacht.

Die einführende Übersicht zur Adressierung in der einsprachigen Lexikographie sei hier nicht ohne den Hinweis abgebrochen, daß aus Platzgründen u.a. (i) zahlreiche Detailprobleme nicht berücksichtigt wurden, daß (ii) eine Analyse eines Artikels mit standardisierter und nichtstandardisierter nichtlemmatischer Adressierung nicht vorgenommen wurde, daß (iii) weiterhin die Adressierung in tabellarischen Wörterbuchartikeln (i.S.v. Wiegand 2000d) ausgeblendet wurde und daß schließlich (iv) zur Adressierung bei Verweisangaben nur einige Hinweise gegeben wurden.

\section{Adressierung in der zweisprachigen Lexikographie}

In einem zweisprachigen Wörterbuchartikel kommt es wesentlich darauf an, $\mathrm{da} ß$ wenigstens eine Aqquivalentbeziehung vom Lemmazeichen der lexikographischen Ausgangssprache (A) zu einer (möglichst lexikalisierten) Einheit der 
Abb. 16: Strukturgraph mit vereinfachter Etikettierung zur (abstrakten und zur isomorphen konkreten) hierarchischen einfachen integrierten Angabestruktur mit vollständiger lemmatischer Adressierung, die wa ${ }_{3}$ aufweist

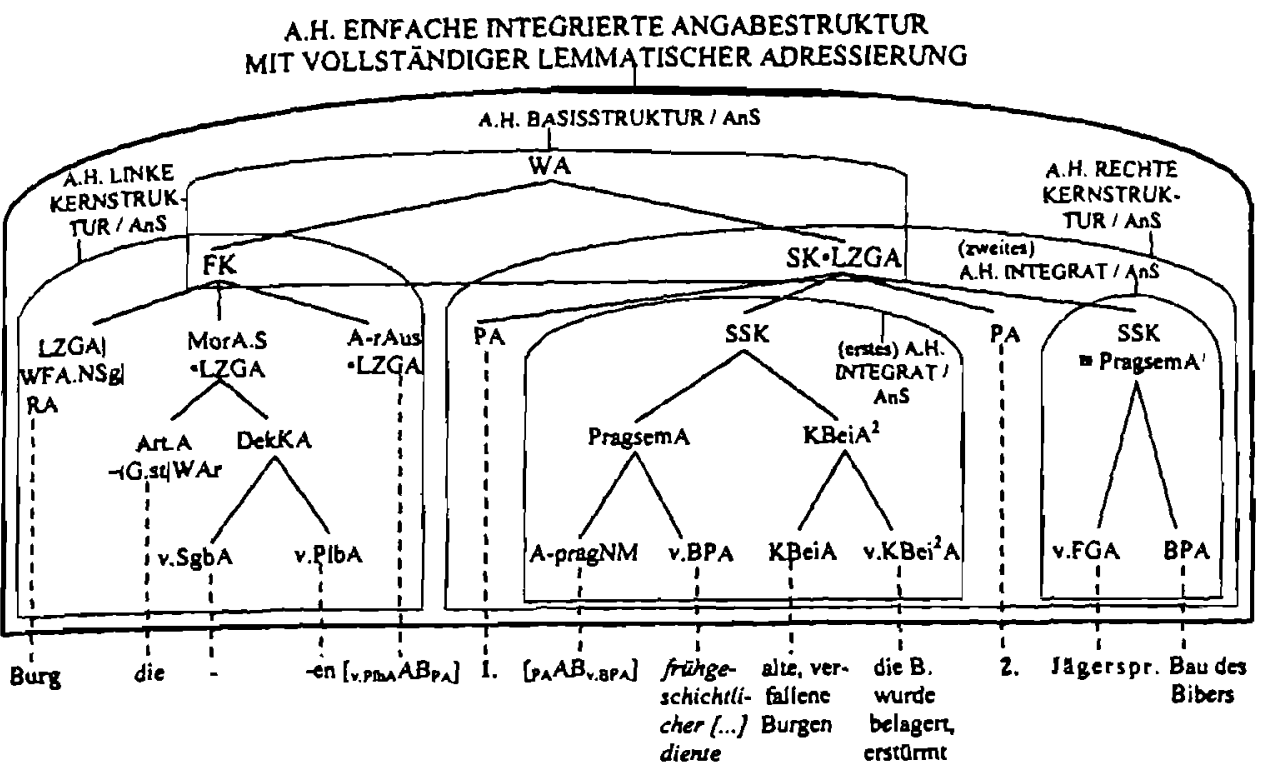

lexikographischen Zielsprache (Z) etabliert wird. Die Äquivalentbeziehung kann mit dem zweistelligen Relationsterm $R_{a ̈}=x$ ist äquivalent mit $y$ benannt werden, wobei " $x$ " eine Variable für ein ausgangssprachliches Lemmazeichen und " $y$ ” eine Variable für ein zielsprachliches Äquivalent ist. In vielen einfachen kleineren zweisprachigen Wörterbüchern (wie z.B. Küen 1993; Prinsloo und Sathekge 1996) gibt es Hunderte von Wörterbuchartikeln, zu welchen der Volltext (neben dem Volltexttitel) aus gerade einem Satz besteht, der durch die Belegung der Variablen in $R_{\ddot{a}}$ gebildet werden kann. Solche Wörterbuchartikel sind 2.B. wa ${ }_{6}$ bis wa $_{15}$ aus Prinsloo und Sathekge (1996) in Abb. 17.

Abb. 17: $w_{6}$ bis $w a_{15}$ aus Prinsloo und Sathekge (1996)

$\begin{array}{llllll}\text { wa } & \text { aim } & \text { lebanya } & w a_{11}: & \text { boy } & \text { mosimane } \\ \text { wa: } & \text { air } & \text { môya } & w a_{12}: & \text { brandy } & \text { poranti } \\ \text { wa. } & \text { altar } & \text { altare } & w a_{13}: & \text { card } & \text { karata } \\ \text { wag: } & \text { arrest } & \text { swara } & w a_{14}: & \text { professor } & \text { moprôfêsa } \\ \text { wa } & \text { bathroom } & \text { bohlapêlô } & w a_{15}: & \text { pronoun } & \text { lešala }\end{array}$

Der genannte Volltextsatz zu wa 6 lautet:

[6] aim ist äquivalent mit lebanya. 
Durch Textverdichtung wird nach dem in 2.2 erläuterten Verfahren aus [6] der zweisprachige Artikel $w_{6} ;$ für die anderen Beispiele gilt dies entsprechend. Zweisprachige Bearbeitungseinheiten wie beispielsweise <aim, lebanya>, $<$ card, karata $>$ sind in zweisprachigen Wörterbuchartikeln unverzichtbar. Auf ein 2-Tupel wie <aim, lebanya> als Element der Bearbeitungsrelation trifft der Relationsterm $\mathrm{R}_{\mathrm{b}}=x$ ist bearbeitet durch y $\mathrm{zu}$, so daß gilt:

(6') aim ist bearbeitet durch lebanya.

Mit aim in (6') ist nicht die Lemmazeichengestaltangabe aim gemeint, denn es ist nicht die LZGA, die in wa 6 mit "lebanya” bearbeitet ist, sondern das Lemmazeichen aim und mit ihm das gesamte Lemmazeichenparadigma (also der Adressat). Auf <aim, lebanya> trifft weiterhin der Relationsterm $R_{\mathrm{kAD}}=y$ ist die Adresse zu $x \mathrm{zu}$, so daß gilt:

(6") aim ist die Adresse zu lebanya.

Im Unterschied zu (6') ist mit aim in (6") die Lemmazeichengestaltangabe gemeint. Der Sachverhalt, daß das 2-Tupel <aim, lebanya> (und entsprechend alle anderen 2-Tupel) sowohl ein Element der Bearbeitungsrelation als auch ein Element der konversen Relation zur Adressierungsrelation sein kann (bzw. sein können), hat zu erheblichen Konfusionen geführt (wie man z.B. bei Hausmann und Werner 1991 sehen kann).

Klar ist dagegen, daß in wa die Wortäquivalentangabe "lebanya" an die Lemmazeichengestaltangabe aim adressiert ist, so daß mit $w_{6} a_{6}$ gerade das ausgesagt wird, was mit [6] auch ausgesagt wird, nämlich der propositionale Gehalt $\mathrm{p}=$ „daß aim und lebanya äquivalent sind".

Wörterbuchartikel wie $w_{6}$ bis $w a_{15}$ wollen nur eine erste Orientierung geben. Sie erwecken allerdings den falschen Eindruck, als gelte die behauptete Äquivalentbeziehung für alle Fälle des Gebrauchs von aim, air usw. Erfahrungsgemäß ist dies aber nicht der Fall. Vielmehr wirken sich ko- und kontextuelle Bedingungen auf die Äquivalentbeziehung aus. Die meisten Äquivalentbeziehungen gelten daher bedingt (= eingeschränkt durch Bedingungen) und nicht unbedingt (= uneingeschränkt), wie man nach $w a_{6}$ bis $w a_{15}$ glauben könnte.

Damit ist der erste wichtige Sachverhalt angesprochen, der dafür verantwortlich ist, daß die Adressierungsbeziehungen selbst in kurzen zweisprachigen Wörterbuchartikeln relativ komplex und z.T. schwer durchschaubar sein können. Denn um eine Äquivalentbeziehungen eindeutig identifizieren zu können, müssen sowohl beim ausgangssprachlichen Lemmazeichen als auch beim zielsprachlichen Äquivalent bestimmte Eigenschaftsausprägungen berücksichtigt werden; dies bedeutet, $\mathrm{da} B$ gegebenenfalls $A$-intern lemmatisch adressierte und Z-intern nichtlemmatisch adressierte Angaben zu diesen Eigenschaftsausprägungen erforderlich werden. Mit solchen Angaben werden 
z.T. auch Bedingungen gemacht, unter denen die Äquivalentbeziehung gilt. Daraus ergibt sich: Im Wörterbuchartikel müssen wenn-dann-Beziehungen, die im Wörterbuchgegenstandsbereich gegeben sind, lexikographisch vertextet sein, so da $B$ bestimmte Angaben nur korrekt sind, wenn andere Angaben korrekt sind. Dieser Zusammenhang wirkt sich auch auf die Adressierung und ihr theoretisches Verständnis aus, was nun am Beispiel von wa ${ }_{16}$ in Abb. 18 erläutert werden soll.

Abb. 18: $w a_{16}$ aus Duden-Oxford (1990)

bestatten [bE'Statn] $t r . V$. (geh.) inter

(formal); bury: bestattet werden be laid to

rest

Im Anschluß an Hausmann und Werner (1991: 2732) weist wa ${ }_{16}$ folgende Bearbeitungseinheiten auf:

Zweisprachige Bearbeitungseinheiten

<bestatten, inter>

$<$ bestatten, bury>

$<$ bestattet werden, be laid to rest>

(2) Einsprachige Bearbeitungseinheiten

(2.1) Ausgangssprachliche Bearbeitungseinheiten

<bestatten, bE'Statn>

<bestatten, tr. V.>

$<$ bestatten, geh.>

(2.2) Zielsprachliche Bearbeitungseinheit

<inter, formal>

In $w a_{16}$ finden sich mithin sieben Bearbeitungseinheiten, so da $\beta$ die auf der Menge aller Angaben von wa ${ }_{16}$ definierte Bearbeitungsrelation sieben 2-Tupel als Elemente aufweist. Entsprechend weist nun die Adressierungsrelation für $w_{16}$ - sie heiße $R_{\text {Ad }}$ - sieben 2-Tupel auf und kann wie folgt extensional bestimmt werden:

$R_{A d}=$ < <inter, bestatten>, <bury, bestatten>, <be laid to rest, bestattet wer-

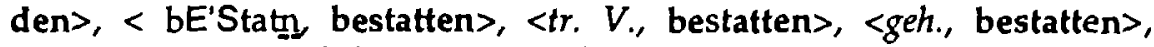
$<$ formal, inter $>$ |. Auf alle 2-Tupel trifft der Relationsterm $x$ ist adressiert an y zu.

Um die Darstellung nicht $z u$ überlasen, berücksichtigen wir im folgenden nur folgenden Ausschnitt von $\mathrm{wa}_{16}$ :

$\mathbf{e}_{4}:$ bestatten $[\ldots]$ tr. V. (geh.) inter (formal) $[\ldots]$ 
$\mathrm{Zu}$ diesem Ausschnitt bilden wir nun folgenden partiellen Volltext $\left(p \mathrm{pt}_{2}\right)$ :

$\mathrm{pVt}_{2}:\left[\mathrm{p}_{1}\right]$ bestatten ist ein transitives Verb.

$\left[\mathrm{p}_{2}\right]$ bestatten wird gehoben verwendet.

$\left[\mathrm{p}_{3}\right]$ inter wird offiziell $(\approx$ formal $)$ verwendet.

[q] inter ist Wortäquivalent zu bestatten.

$\mathrm{pVt}_{2}$ besteht aus vier Aussagen, wobei $\mathrm{q}$ die Aussage über die Äquivalentbeziehung ist; $q$ gilt aber nur dann, wenn $p_{1}, p_{2}$ und $p_{3}$ gelten. Wir können mithin feststellen: Wenn bestatten ein transitives Verb ist, und wenn es gehoben verwendet wird, und wenn inter offiziell verwendet wird, dann sind bestatten und inter Wortäquivalente. In Abb. 19 werden nun die Adressierungsbeziehungen in $e_{4}$ veranschaulicht.

Abb. 19: Veranschaulichung zu einigen Adressierungsbeziehungen in $\mathbf{e}_{4}$

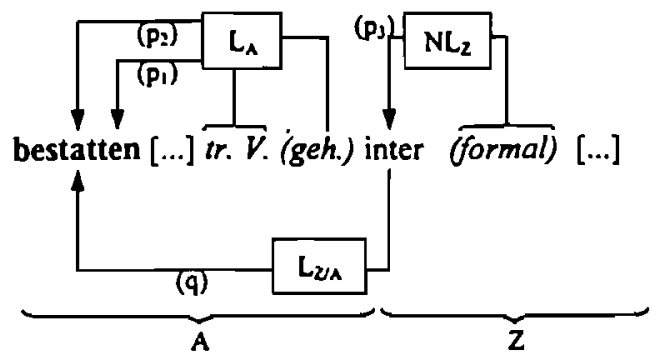

Abkürzungen: $\mathrm{A}=$ lexikographische Ausgangssprache (hier: $\mathrm{Dt}$.); $\mathrm{Z}=$ lexikographische Zielsprache (hier: Engl.); $\mathrm{L}_{\mathrm{A}}=\mathrm{A}$-intem lemmatisch adressier; $\mathrm{NL}_{\mathrm{Z}}=\mathrm{Z}$-intern nichtlemmatisch adressiert; $L_{2 / A}=$ von $Z$ nach $A$ lemmatisch adressiert; Erläuterungen:,$\longrightarrow u \longrightarrow y^{\prime \prime}$ bedeutet soviel wie $x$ ist artikelintern $u$-adressiert an y mit „u“ als Variable fur $\mathrm{L}_{A}, \mathrm{NL}_{2}$ und $\mathrm{L}_{\text {ZA }}$.

Wir können nun feststellen: Daß die Adressierung von „inter" an „bestatten” (also die lemmatische Adressierung von $Z$ nach $A$ ) korrekt ist, hat zur Bedingung, daß (i) die Adressierung von "tr. V." und von "geh." an bestatten (also die A-interne lemmatische Adressierung) korrekt ist und weiterhin, daß (ii) die Adressierung von "formal" an "inter" (also die'Z-interne nichtlemmatische Adressierung) korrekt ist.

Damit können wir folgende beiden Arten von Adressierungsbeziehungen unterscheiden:

- bedingende Adressierungsbeziehungen, und

- bedingte Adressierungsbeziehungen. 
Die Adressierungsbeziehung "inter ist von $Z$ nach $A$ lemmatisch an bestatten adressiert" ist eine bedingte Adressierungsbeziehung. Alle anderen Adressierungsbeziehungen in $\mathrm{e}_{4}$ sind bedingende Adressierungsbeziehungen.

Alle Adressierungsbeziehungen in $w a_{6}$ bis $w a_{15}$ (vgl. Abb. 17) sind dagegen unbedingte Adressierungsbeziehungen, weil keine ausgangssprachlichen und/ oder zielsprachlichen Bedingungen dafür angegeben sind, daß die Äquivalentbeziehungen korrekt sind. Auch in einsprachigen Wörterbuchartikeln - das sei hier ergänzend zu den Ausführungen in 2. erwähnt - können bedingte und bedingende Adressierungsbeziehungen auftreten. Dies sei nachfolgend kurz anhand des HWDG-Artikels wa ${ }_{17}$ in Abb. 20 erläutert.

Abb. 20: $w_{17}$ aus dem HWDG

Moos, das; -es, -e 1. kleine, in großer, dicht stehender Menge, vorzugsweise an schattigen, feuchten Stellen wachsende immergrüne, sich durch Sporen vermehrende Pflanze: weiches, grünes M.; Moose und Flechten; sich im Wald ins M., auf das $M$. setzen - 2. 1o. Pl./ u mg. Geld (1): da muß doch eine Masse $M$. herausspringen

Im zweiten semantischen Subkommentar von wa ${ }_{17}$ gelten u.a. folgende Adressierungsbeziehungen:

(i) Geld ist lemmatisch an Moos adressiert.

(ii) "O. Pl." ist lemmatisch an Moos adressiert.

(iii) "umg." ist lemmatisch an Moos adressiert.

Die Bedingungsverhältrisse werden anhand folgender Aussagen deutlich:

(a) Wenn Moos soviel bedeutet wie Geld, dann wird Moos ohne Plural und umgangssprachlich verwendet.

(b) Wenn Geld an Moos adressiert ist, dann sind "o. Pl." und „umg." an Moos adressiert.

Die Adressierungsbeziehung (i) ist daher bedingend; die Adressierungsbeziehungen (ii) und (iii) sind bedingt. ${ }^{7}$

Ein zweiter Sachverhalt, warum Adressierungsbeziehungen in zweisprachigen Wörterbüchern komplex sind, besteht darin, daß es zu einem ausgangssprachlichen Lemmazeichen mehrere zielsprachliche Äquivalente geben kann, so daß mit Äquivalentunterscheidungsangaben ihre Bedeutungsunterschiede angegeben werden müssen. Wie die Adressen von Äquivalentunterscheidungsangaben sind, ist vom Benutzer häufig schwer zu ermitteln (vgl. Meyer und Wiegand 2000). Auch eine saubere (vgl. $\mathrm{wa}_{4}, 2.3$ !) theoretische 
Lösung macht große Schwierigkeiten. Die folgende partielle Analyse anhand von wa ${ }_{18}$ in Abb. 21 ist daher als Versuch einzuschätzen (vgl. auch Wiegand 2000e mit weiteren Versuchen).

Abb. 21: $w a_{18}$ aus Slabý et al. 1994

blau adj azul || [hell-] celeste, (poet) cerúleo \| [Heraldik] azur $\|$ [Auge \&] garzo \| [vor Kălte] livido, amoratado $\|$ [Traube] tinto $\|$ (fig) [betrunken] borracho $\| 0 \sim$ anlaufen lassen [Stahl] pavonar $\| \sim$ färben teñir de azul od antil, azular $\| \sim$ unterlaufene Augen ojos mpl lividos \|l [Augenringe] ojerds fpl

Die Äquivalentunterscheidungsangaben ( $\ddot{U} U n t A$ ) in $w_{18}$ sind daran erkennbar, da $\beta$ sie in eckigen Klammern stehen. Äquivalentunterscheidungsangaben können unterschiedlich realisiert sein, z.B. als Fachgebietsangabe (wie „Heraldik") oder als Bezugsobjektangabe (wie "Traube"). Meistens sind sie Angaben aus anderen Angabeklassen in äquivalentunterscheidender Funktion oder sie werden vom Lexikographen ad hoc gebildet. In wa ${ }_{18}$ unterscheiden sie für den deutschen Benutzer $n$ (mịt $n \geq 2$ ) in Äquivalentangaben erwähnte spanische Äquivalente zu einem deutschen Lemmazeichen.

Ich argumentiere nachfolgend anhand folgenden Ausschnittes aus wa ${ }_{18}$ :

$$
\mathrm{e}_{5}: \text { blau [...] [Traube] tinto [...] }
$$

Wenn "Traube" die adressierte Angabe ist, gibt es für die hier fraglichen Adressierungsbeziehungen in $e_{5}$ drei im Prinzip mögliche Fälle:

(1) "Traube" ist nichtlemmatisch nach rechts an die Wortäquivalentangabe tinto adressiert, so daß eine nichtlemmatische Rechtsadressierung von der Ausgangs- in die Zielsprache vorliegt.

(2) "Traube” ist lemmatisch nach links an die Lemmazeichengestaltangabe blau adressiert, so daß eine lemmatische ausgangsspracheninterne $(=\mathrm{A}$ interne) Linksadressierung gegeben ist.

(3) "Traube" ist sowohl an blau als auch an tinto adressiert. In diesem Falle hätte man eine „Doppeladressierung” nach (1) und (2).

Eine Veranschaulichung der Adressierungsbeziehung in $e_{5}$ nach Fall (1) findet sich in Abb. 22.

Mit Bezug auf den Wörterbuchgegenstandsbereich von Slabý et al. (1994) (also Deutsch und Spanisch) kann $e_{5}$ (Fall 1) wie folgt interpretiert werden: Wenn tinto auf das Bezugsobjekt Traube(n) bezogen ist, dann ist das Wortäquivalent zu tinto das Lemmazeichen blau. Dem entspricht auf der Ebene der Wörterbuchform: Wenn "Traube" an tinto adressiert ist, dann ist tinto an blau 
Abb. 22: Erste Veranschaulichung zu den Adressierungsbeziehungen in $e_{5}$ (Fall 1)

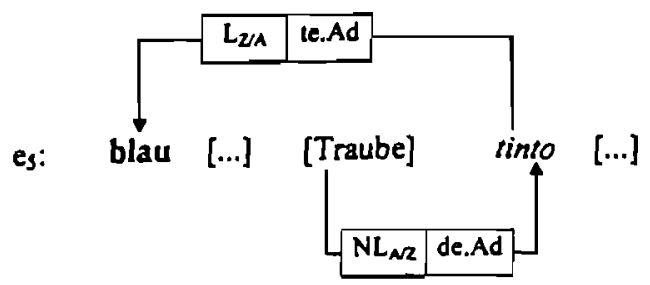

Abkürzungen: te. $A d=$ bedingte Adressierung; de. $A d=$ bedingende Adressierung; $\mathrm{L}_{\mathrm{ZA}_{\mathrm{A}}}=$ von der Zielsprache $(Z)$ nach der Ausgangssprache $(A)$ lemmatisch adressiert; $N L_{N Z}=$ von $A$ nach $Z$ nichtlemmatisch adressiert. Erläuterungen: , $x \longrightarrow$ u $v \longrightarrow y^{\prime \prime}$ bedeutet soviel wie $x$

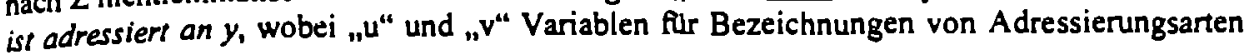
sind.

adressiert. Oder anders ausgedrückt: Wenn tinto Adresse von „Traube” ist, dann ist blau Adresse von tinto.

Eine Veranschaulichung der Adressierungsbeziehung in $e_{5}$ nach Fall (2) findet sich in Abb. 23.

Abb. 23: Zweite Veranschaulichung zu den Adressierungsbeziehungen in $e_{5}$ (Fall 2)

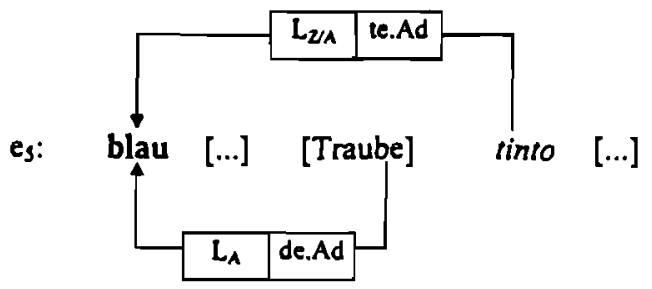

Im Hinblick auf den Wörterbuchgegenstandsbereich kann $e_{5}$ (Fall 2) wie folgt gedeutet werden: Wenn blau auf das Bezugsobjekt Traube( $n$ ) bezogen ist, dann ist tinto ein Wortäquivalent zu blau. Dem entspricht auf der Ebene der Wörterbuchform: Wenn "Traube" an blau adressiert ist, dann ist auch tinto an blau adressiert. Oder anders gesagt: Wenn blau die Adresse von "Traube” ist, dann ist blau auch die Adresse von tinto.

Der 3. Fall wäre eine Kombination der beiden ersten Fälle und seine Interpretation entsprechend wenig sinnvoll.

Damit haben wir nun das große Rätsel, welche der beiden Interpretationen nach welchem Kriterium als die angemessene gelten kann. Das Kriterium kann nur der genuine Zweck eines zweisprachigen Wörterbuchs sein (vgl. Wiegand 1996: 42ff.) und damit die für das Wörterbuch vorgesehenen Wörterbuchfunk- 
tionen (i.S.v. Tarp 1994). In Slabý et al. (1994) findet man dazu allerdings nichts Nennenswertes. Daher argumentiere ich im folgenden $z w a r$ anhand von $\mathrm{wa}_{18}$ aber ohne Bezug zu den Umtexten von Slabý et al. (1994); dies ist deswegen vertretbar, weil es hier um systematische Zusammenhänge geht und nicht um ein bestimmtes zweisprachiges Wörterbuch. ${ }^{8}$

Wir betrachten im folgenden drei Fälle.

Fall a: $\mathrm{wa}_{18}$ steht in einem Wörterbuch $\mathrm{Wb}_{1}$, für das gilt:

(i) $\mathrm{Wb}_{1}$ ist monoakzessiv.

(ii) $\mathrm{Wb}_{1}$ ist für deutsche Benutzer konzipiert.

(iii) $\mathrm{Wb}_{1}$ ist ein sog. aktives Wörterbuch, so daß die Produktions- und die Hinübersetzungsfunktion als primäre Wörterbuchfunktionen gelten.

Angenommen, ein Deutscher möchte den Satz

(7) Blaue Trauben passen gut zum Käse

ins Spanische übersetzen, weiß aber nicht, was blau auf Spanisch heißt und schlägt in $\mathrm{Wb}_{1}$ nach, dann muß er $e_{5}$ lesen wie: Wenn blau auf Trauben bezogen ist, dann heißt es auf Spanisch tinto. Damit gilt für Fall a die zweite Interpretation der Adressierungsbeziehungen.

Fall b: $\mathrm{wa}_{18}$ steht in einem Wörterbuch $\mathrm{Wb}_{2}$, für das gilt:

(i) $\mathrm{Wb}_{2}$ ist monoakzessiv.

(ii) $\quad \mathrm{Wb}_{2}$ ist für spanische Benutzer konzipiert.

(iii) $\mathrm{Wb}_{2}$ ist ein sog. passives Wörterbuch, so daß die Rezeptions- und die Herübersetzungsfunktion als primäre Wörterbuchfunktionen gelten.

Will ein Spanier (7) ins Deutsche übersetzen und weiß nicht, was blau bedeutet, dann gilt die gleiche Interpretation wie in Fall a. (Es ist klar, daß in einem Wörterbuch für spanische Benutzer die Äquivalentunterscheidungsangabe in Spanisch stehen müßte.)

Fall c: $\mathrm{wa}_{18}$ steht in einem Wörterbuch $\mathrm{Wb}_{3}$, für das gilt:

(i) $\mathrm{Wb}_{3}$ ist extern polyakzessiv (i.S.v. Wiegand 1996: 47) und weist ein span.-dt. alphabetisches Äquivalentregister auf mit Registereinträgen wie "tinto blau".

(ii) $\quad \mathrm{Wb}_{3}$ ist primär für deutsche und sekundär für spanische Benutzer konzipiert.

(iii) $\mathrm{Wb}_{3}$ ist in erster Linie ein aktives Wörterbuch für Deutsche (wie $\mathrm{Wb}_{1}$, (iii)) und in zweiter Linie ein aktives Wörterbuch für Spanier.

Will ein Spanier in einem spanischen Text, in dem tinto auf das bezogen ist, was Deutsche Trauben nennen, ins Deutsche übersetzen und kennt kein deut- 
sches Äquivalent, dann kann er zunächst im Register von $\mathrm{Wb}_{3}$ nachschlagen und dann unter blau. Für diesen Fall gilt offensichtlich die erste Interpretation.

In den Benutzungshinweisen eines zweisprachigen Wörterbuchs vom Typ $\mathrm{Wb}_{1}$ muß demnach ein Wörterbucheintrag wie $e_{5}$ wie folgt erläutert werden:

Wenn Blau sich auf Trauben bezieht, dann heißt es auf Spanisch tinto. In einem Wörterbuch vom Typ $\mathrm{Wb}_{2}$ muß das gleiche auf Spanisch stehen. Schließlich muß in den Benutzungshinweisen eines Wörterbuchs vom Typ $\mathrm{Wb}_{3}$ für die deutschen Benutzer das gleiche stehen wie in $\mathrm{Wb}_{1}$, und für die spanischen Benutzer, die über das erweiterte. Äquivalentregister zugreifen möchten, muß es (auf Spanisch) heißen: Wenn tinto auf Trauben bezogen ist, heißt es auch Deutsch blau.

Die gerade gegebenen pauschalen Hinweise zu den Benutzungshinweisen in zweisprachigen Wörterbüchern sind zu verstehen als ein Plädoyer, die Adressierungsbeziehungen anhand von Beispielen für Wörterbucheinträge und durch die Wiedergabe der wenn-dann-Beziehungen zu erklären. Der derzeitige Zustand, $d a ß$ in vielen zweisprachigen Wörterbüchern die Adressierungsbeziehungen meistens nicht erläutert werden, ist - so sollte nach den Ausführungen in diesem Beitrag wohl deutlich geworden sein - nicht im Sinne der Benutzer.

\section{Ausblick}

Hochverdichtete und stark standardisierte Wörterbuchartikel in ein- und zweisprachigen Wörterbüchern stellen keine natürlichsprachlichen Texte dar (vgl. auch näher Wiegand 2000f.). Sie bilden vielmehr ein hochkomplexes Ineinander von formaler Syntax und der Semantik derjenigen Sprach(en), die den Wörterbuchgegenstandsbereich bilden. Die Syntax hat zwei Aufgaben: sie ordnet die Konstituenten (Angaben) linear in eine bestimmte Reihenfolge und sie stellt Beziehungen zwischen den Konstituenten her. Letzteres wird als Adressierung analysiert. $\mathrm{Da}$ immer vorausgesetzt werden muß, daß der Benutzer das, was er sucht, nicht kennt und weiterhin, daß er ganze Teile der lexikographisch bearbeiteten Sprache nicht kennt, so daß er die Konstituenten nicht aufgrund ihrer Bedeutungen aufeinander beziehen kann, muß der Artikeltext so gestaltet sein, daß man nicht nur erkennen kann, welche Artikelpositionen aufeinander folgen und beim suchenden Lesen gerade gegeben ist, sondern es muß anhand von Eigenschaften der Artikelform auch jeweils klar sein, an welche Adresse eine Angabe adressiert ist, weil sonst nicht sichergestellt ist, $\mathrm{da} B$ ein Benutzer lexikographische Informationen richtig erschließen kann. Dies gilt besonders für allgemeine zweisprachige Wörterbücher. Das heißt, da $B$ in den Benutzungshinweisen nicht nur die mikrostrukturelle Konstituenz von Wörterbuchartikeln, sondern auch die Angabestruktur und damit die Adressierung erläutert werden muß. Es ist daher ein Desiderat, herauszufinden, wie dies am einfachsten geschehen kann. 


\section{Endnoten}

1. Es ist geplant, in dem mehrsprachigen Wörterbuch zur Lexikographie und Wörterbuchforschung/Dictionary of Lexicography and Dictionary Research (das voraussichtlich herausgegeben wird von Rufus H. Gouws, Henning Bergenholtz, Matthias Kammerer, Angelika Storrer, Sven Tarp, Herbert Ernst Wiegand und Werner Wolski) die metalexikographische Terminologie und lexikographische Werkstattsprache möglichst exhaustiv zu bearbeiten. Die derzeit noch vorläufige Lemmakandidatenliste enthält für den Bereich der Adressierung folgende Lemmakandidaten: Adresse; Adresse, artikelinteme/artikelexterne/nichtstandardisierte/standardisierte/wörterbuchexterne; Adressenfundort; Adressensymbol; Adressierung: Adressierung bedingte/bedingende/unbedingte/ infralemmatische/lemmatische/ nichtlemmatische/sublemmatische/nichtstandardisierte/standardisierte; Adressierungsrelation; Adressierungsstruktur, Außentextadresse, Nahadressierung, Femadressierung, Glossat, Glossatadresse, Präglossat, Binnenglossat, Postglossat, Umtextadresse, Verweisadresse, Verweisaußenadresse. Die meisten dieser Termini (und einige weitere) werden nachfolgend erläutert.

2. Die zentralen Teile einer Theorie der Wörterbuchform liegen inzwischen vor; vgl. u.a. Wiegand (1988, 1989, 1989a, 1989b, 1990, 1991, 1993 [1994], 1995, 1995a, 1996, 1996a, 1996b, 1996c, 1997, 1998, 1998a, 1998b, 1999, 1999a, 2000, 2000b, 2000d, 2000e); Bergenholtz, Tarp und Wiegand (1999); Kammerer und Wiegand (1998); Konerding und Wiegand (1995); Meyer und Wiegand (2000); vgl. auch: http://www.uni-heidelberg.de/institute/fak9/gs/sprache2/ hew_publ.htm.

3. Vgl. die in Anm. 2 genannte Literatur.

4. Für eine durchgehende Erläuterung aller Segmentationsschritte einer funktional-positionalen Segmentation vgl. z.B. Wiegand 2000: 235ff.

5. Zu weiteren Eigenschaften, die den Sonderstatus der Lemmazeichengestaltangabe begründen, vgl. Wiegand 2000e.

6. Mit den Glossaten sind eine Reihe von Segmentationsproblemen verbunden. Diese lassen sich wahrscheinlich lösen, wenn das in Meyer und Wiegand (2000: 147) angedeutete Konzept der gebundenen Angaben erweitert und präzisiert wird, was hier nicht möglich ist.

7. Mit der Festlegung der Bedingungsverhältrisse sind sprachtheoretische Voraussetzungen gegeben, worauf hier nicht näher eingegangen werden kann.

8. Zu Slabý et al. (1994) kann im übrigen Meyer und Wiegand (2000: 114ff.) verglichen werden.

\section{Literatur}

Bergenholtz, H. und S. Tarp (Red.). 1995. Manual of Specialised Lexicography. The Preparation of Specialised Dictionaries. Mit Beiträgen von G. Duvd, A.-L. Laursen, S. Nielsen, O. NorlingChristensen und J. Pedersen. Amsterdam/Philadelphia: John Benjamirs.

Bergenholtz, H., I. Contell et al. 1997. Nordisk Leksikografisk Ordbok. Mit Beiträgen von H. Haraldsson, H.K. Mikkelsen und J. Sivula. Skrifter utgitt av Nordisk forening for leksikografi. skrift nr. 4. Oslo: Universitetsforlaget AS.

Bergenholtz, H., S. Tarp und H.E. Wiegand. 1999. Datendistributionsstrukturen, Makro- und Mikrostrukturen in neueren Fachwörterbüchern. HSK 14.2: 1762-1832. 
Burkhanov, I. 1998. Lexicography. A Dictionary of Basis Terminology. Rzeszów: Wyzszej szhoty pedagogicznej.

BW = Brockhaus-Wahrig: Deutsches Wörterbuch in sechs Bänden. 1980-1984. Hrsg. v. Gerhard Wahrigt, Hildegard Krämer und Harald Zimmermann. 1. Bd.: A-BT 1980. 2. Bd.: BU-FZ 1981. 3. Bd.: G-JZ 1981. 4. Bd.: K-OZ 1982. 5. Bd.: P-STD 1983. 6. Bd.: STE-ZZ 1984. Wiesbaden: Brockhaus. Campe-WdS = Joachim Heinrich Campe. 1969. Wörterbuch der Deutschen Sprache. I. A-E. Mit einer Einführung und Bibliographie von Helmut Henne. Documenta Linguistica. Reihe II. Wörterbücher des 17. und 18. Jahrhunderts. Hildesheim/New York. [Reprogr. Nachdruck der Ausgabe Braunschweig 1807].

Duden-Oxford $1990=$ Duden-Oxford. Großwörterbuch Englisch. Englisch-Deutsch/Deutsch-Englisch. Hrsg. v. der Dudenredaktion und Oxford University Press. Red. Leitung: Werner ScholzeStubenrecht und John Sykes. Maanheim [...]: Dudenverlag 1990.

Glück, H. (Red.). 2000. Metzler Lexikon Sprache. 2. überarb. und erw. Aufl. mit 70 Abbildungen, davon 17 Karten. Stuttgart/Weimar: J.B. Metzler.

Hartmann, R.R.K. und G. James. 1998. Dictionary of Lexicography. London/New York: Routledge.

HSK 5.1 = Wörterbücher. Ein internationales Handbuch zur Lexikographie/Dictionaries. An International Encyclopedia of Lexicography/Dictionnaires. Encyclopédie internationale de lexicographie. Erster Teilbd. 1989. Hrsg. v. Franz Josef Hausmann, Oskar Reichmann, Herbert Emst Wiegand und Ladislav Zgusta. Handbücher zur Sprach- und Kommunikationswissenschaft 5.1. Berlin/ New York: De Gruyter.

HSK 5.2 = Wörterbücher. Ein internationales Handbuch zur Lexikographie/Dictionaries. An In!ernational Encyclopedia of Lexicography/Dictionnaires. Encyclopédie internationale de lexicographie. Zweiter Teilbd. 1990. Hrsg. v. Franz Josef Hausmann, Oskar Reichmann, Herbert Emst Wiegand und Ladislav Zgusta. Handbücher zur Sprach- und Kommunikationswissenschaft 5.2. Berlin/ New York: De Gruyter.

HSK 5.3 = Wörterbücher. Ein internationales Handbuch zur Lexikographie/Dictionaries. An International Encyclopedia of Lexicography/Dictionnaires. Encyclopédie internationale de lexicographie. Dritter Teilbd. 1991. Hrsg. v. Franz Josef Hausmann, Oskar Reichmann, Herbert Emst Wiegand und Ladislav Zgusta. Handbücher zur Sprach- und Kommunikationswissenschaft 5.3. Berlin/ New York: De Gruyter.

HSK 14.2 = Fachsprachen. Ein internationales Handbuch zur Fachsprachenforschung und Terminologiewissenschaft/Languages for Special Purposes. An Intemational Handbook of Special-Language and Terminology Research. 2. Halbbd. 1999. Hrsg. v. Lothar Hoffmann, Hartwig Kalverkämper und Herbert Emst Wiegand. In Verbindung mit Christian Galinski und Werner Hüllen. Handbücher zur Sprach- und Kommunikationswissenschaft 14.2. Berlin/New York: De Gruyter.

HWDG = Handwörterbuch der deutschen Gegenwartssprache. In zwei Bänden. 1984. Von einem Autorenkollektiv unter der Leitung v. Günter Kempcke [...]. Bd. 1: A-K. Bd. 2: L-Z. Berlin: Akademie Verlag.

Kammerer, M. und H.E. Wiegand. 1998. Uber die textuelle Rahmenstruktur von Printwörterbüchem. Präzisierungen und weiterführende Überlegungen. Lexicographica 14: 224-238.

Kluge, F. 1995. Etymologisches Wörterbuch der deutschen Sprache. Bearb. v. Elmar Seebold. 23. erw. Aufl. Berlin/New York: De Gruyter.

Küen, E. 1993. Deutsch-Niederlandisches Lernwörterbuch. Hamburg: Buske.

Landau, S.I. 1984. Dictionaries. The Art and Craft of Lexicography. New York: Cha rles Scribner's Sons. 
Martínez de Sousa, J. 1995. Diccionario de lexicografía práctica. Barcelona: Bibliograf, S.A.

Meyer, M. und H.E. Wiegand. 2000. Gemischt-semiintegrierte Mikrostrukturen für deutsch-spanische Printwörterbücher. Wiegand, H. E. (Red.). 2000. Studien zur zweisprachigen Lexikographie mit Deutsch V: 87-171. Germanistische Linguistik 151-152. Hildesheim/New York: Olms.

Pan Zaiping und H.E. Wiegand. 1995. Über die Musterartikel für das Große Deutsch-Chinesische Wörterbuch. Zugleich ein Beitrag zu einer Theorie zweisprachiger lexikographischer Texte. Wiegand, H. E. (Red.). 1995. Studien zur zweisprachigen Lexikographie mit Deutsch II: 63-190. Germanistische Linguistik 127-128. Hildesheim/New York: Olms.

Prinsloo, D.J. und B.P. Sathekge. 1996. New Sepedi Dictionary. English-Sepedi (Northern Sotho); Sepedi (Northern Sotho)-English. Pietermaritzburg/Kapstadt/Randburg: Shuter \& Shooter.

Rey-Debove, J. 1971. Etude linguistique et sémiotique des dictionnaires français contemporains. Approaches to Semiotics 13. Den Haag/Paris: Mouton.

Slabý, R.J., R. Grossmann und C. lllig. 1994. Wörterbuch der spanischen und deutschen Sprache. 4. Aufl. Wiesbaden: Horrasowitz.

Svensén, B. 1993. Practical Lexicography. Principles and Methods of Dictionary-Making. Aus dem Schwedischen übers. v. John Sykes und Kerstin Schofield. Oxford/New York: Oxford University Press.

Tarp, S. 1994. Funktionen in Fachwörterbüchern. Schaeder, B. und H. Bergenholtz (Red.). Fachlexikographie. Fachwissen und seine Repräsentation in Wörterbüchern: 229-246. Forum für Fachsprachen-Forschung 23. Tübingen: Narr.

Wiegand, H.E. 1988. Wörterbuchartikel als Text. Harras, G. (Red.). 1988. Das Wörterbuch. Artikel und Verveisstrukturen. Jahrbuch 1987 des Instituts für deutsche Sprache: 30-120. Sprache der Gegenwart LXXIV. Düsseldorf: Schwann. [Auch in: Wiegand, H.E. 2000a: 877-950].

Wiegand, H.E. 1989. Aspekte der Makrostruktur im allgemeinen einsprachigen Wörterbuch: alphabetische Anordnungsformen und ihre Probleme. HSK 5.1: 371-409.

Wiegand, H.E. 1989a. Der Begriff der Mikrostruktur: Geschichte, Probleme, Perspektiven. HSK 5.1: $409-462$.

Wiegand, H.E. 1989b. Arten von Mikrostrukturen im allgemeinen einsprachigen Wörterbuch. HSK 5.1: 462-501.

Wiegand, H.E. 1990. Printed Dictionaries and their Parts as Texts. An Overview of More Recent Research as an Introduction. Lexicographica 6: 1-126. [Auch in: Wiegand, H.E. 2000a: 951-1062]

Wiegand, H.E. 1991. Über die Strukturen der Artikeltexte im Frühneuhochdeutschen Wörterbuch. Zugleich ein Versuch zur Weiterentwicklung einer Theorie lexikographischer Texte. Goebel, U. und O. Reichmann (Red.). 1991. Historical Lexicography of the German Language. Vol. 2: 341673. Studies in Russian and German 3. Lewiston/Queenston/Lampeter: Edwin Mellen Press.

Wiegand, H.E. 1993 [1994]. Kritische Lanze für Fackel-Redensartenwörterbuch. Bericht und Diskussion zu einem Workshop in der Osterreichischen Akademie der Wissenschaften am 14.2.1994. Lexicographica 9: 230-271. [Auch in: Wiegand, H.E. 2000a: 1063-1105].

Wiegand, H.E. 1995. Lexikographische Texte in einsprachigen Lemerwörterbüchern. Kritische Überlegungen anläBlich des Erscheinens von Langenscheidts Großwörterbuch Deutsch als Fremdsprache. Popp, H. (Red.). 1995. Deutsch als Fremdsprache. An den Quellen eines Faches. Festschrift für Gerhard Helbig zum 65. Geburtstag: 463-499. München: Iudicium. [Auch in: Wiegand, H.E. 2000a: 1128-1162]. 
Wiegand, H.E. 1995a. Deutsch-Türkmenisches Wörterbuch. Einblicke in die Wörterbucharbeit an der Türkmenischen Staatlichen Magtymguly-Universität in Aschghabat. Lexicographica 10: 249-300.

Wiegand, H.E. 1996. Das Konzept der semiintegrierten Mikrostrukturen. Ein Beitrag zur Theorie 2weisprachiger Printwörterbücher. Wiegand, H.E. (Red.) 1996. Wörterbuicher in der Diskussion II. Vorträge aus dem Heidelberger Lexikographischen Kolloquium: 11-43. Lexicographica. Series Maior 76. Tübingen: Max Nieweyer. [Auch in: Wiegand, H.E. 2000a: 1193-1277)

Wiegand, H.E. 1996a. A Theory of Lexicographic Texts. An Overview. South African Journal of Linguistics 14(4): 134-149.

Wiegand, H.E. 1996b. Deutsch-Usbekisches Wörterbuch. Einblicke in die Wörterbucharbeit an der Staatlichen Usbekischen Weltsprachen-Universität in Taschkent. Lexicographica 12: 190-254. [Auch in: Wiegand, H.E. 2000a: 1339-1409].

Wiegand, H.E. 1996c. Über die Mediostrukturen bei gedruckten Wörterbüchern. Zettersten, A and V. Hjemager Pedersen (Red.). 1996. Symposium on Lexicography VII. Proceedings of the Seventh Symposium on Lexicography May 5-6, 1994 at the University of Copenhagen: 11-43. Lexicographica. Series Maior 76. Tübingen: Niemeyer. [Auch in: Wiegand, H.E. 2000a: 1163-1192].

Wiegand, H.E. 1997. Printed Language Dictionaries and their Standardization: Notes on the Progress toward a General Theory of Lexicography. Hock, H.H. (Red.). 1997. Historical, Indo-European, and Lexicographical Studies. A Festschrift for Ladislav Zgusta on the Occasion of his 70 th Birthday: 319-380. Trends in Linguistics. Studies and Monographs 90. Berlin: Mouton de Gruyter.

Wiegand, H.E. 1998. Wörterbuchforschung. Untersuchungen zur Wörterbuchbenutzung, zur Theorie, Geschichte, Kritik und Automatisierung der Lexikographie. 1. Teilbd. Mit 159 Abbildungen im Text. Berlin/New York: De Gruyter.

Wiegand, H.E. 1998a. Lexikographische Textverdichtung. Entwurf zu einer vollständigen Konzeption. Zettersten, A. und V. Hjomager Pedersen (Red.). 1998. Symposium on Lexicography VIII. Proceedings of the Eighth Symposium on Lexicography May 2-4, 1996 at the University of Copenhagen: 1-35. Lexicographica. Series Maior 90. Tübingen: Niemeyer. [Auch in: Wiegand, H.E. 2000a: 1454-1489].

Wiegand, H.E. 1998b. Altes und Neues zur Makrostruktur alphabetischer Printwörterbücher. Wiegand, H.E. (Red.). 1998. Wörterbücher in der Diskussion III. Vorträge aus dem Heidelberger Lexikographischen Kolloquium: 348-372. Lexicographica. Series Maior 84. Tübingen: Niemeyer. [Auch in: Wiegand, H.E. 2000a: 1428-1453].

Wiegand, H.E. 1999. Artikel einsprachiger Lernerwörterbücher, Textgestaltwahmehmung und Suchbereichstrukturen. Plädoyer für übersichtliche Printwörterbücher im Zeitalter der Neuen Medien. Skibitzki, B. und B. Wotjak (Red.). 1999. Linguistik und Deutsch als Fremdsprache. Festschrift für Gerhard Helbig zum 70. Geburtstag: 259-281. Tübingen: Niemeyer.

Wiegand, H.E. 1999a. Semantics and Lexicography. Selected Studies (1976-1996). Hrsg. v. Antje Immken und Wemer Wolski. Lexicographica. Series Maior 87. Tübingen: Niemeyer.

Wiegand, H.E. 2000. Utber Suchbereiche, Suchzonen und ihre textuellen Strukturen in Printwörterbüchem. Ein Beitrag zur Theorie der Wörterbuchform. Wiegand, H.E. (Red.). 2000. Wörterbücher in der Diskussion IV. Vorträge aus dem Heidelberger Lexikographischen Kolloquium: 233-301. Lexicographica. Series Maior 1000. Tübingen: Niemeyer. 
Wiegand, H.E. 2000a. Kleine Schriften. Eine Auswahl aus den Jahren 1970 bis 1999 in zwei Bänden. Bd. 1: 1970-1988; Bd. 2: 1988-1999. Hrsg. v. Matthias Kammerer und Werner Wolski. Berlin/New York: De Gruyter.

Wiegand, H.E. 2000b. Sprachkontaktwörterbücher: Typen, Funktionen, Strukturen. Petkov, P. und H.E. Wiegand (Red.). Theoretische und praktische Probleme der Lexikographie. 1. Internationales Kolloquium zur Wörterbuchforschung am Institut "Germanicum" der St. Kliment-Ohridski-Universität in Sofia. Germanistische Linguistik. Hildesheim/New York: Olms.

Wiegand, H.E. 2000c. Fremdwörterbücher und Sprachwirklichkeit. Jahrbuch des Instituts für deutsche Sprache. Erscheint.

Wiegand, H.E. 2000d. Über tabellarische Wörterverzeichnisse und deren Wörterbuchartikel. Ein Beitrag zur Theorie der Wörterbuchform. Lexicographica. Erscheint.

Wiegand, H.E. 2000e. Probleme der Adressierung in der zweisprachigen Lexikographie. Vortrag, gehalten auf dem III. Ost-West-Kolloquium im Februar 2000. Erscheint.

Wiegand, H.E. 2000f. Wissen, Wissensrepräsentation und Printwörterbücher. Heid, Ulrich, Stefan Evert, Egbert Lehmans und Christian Rohrer (Red.). 2000. Proceedings of the Ninth EURALEX International Congress, Euralex 2000, Stuttgart, Germany, August 8th-12th, 2000. Vol. 1: 15-38. Stuttgart: Institut für Maschinelle Sprachverarbeitung, Universität Stuttgart.

Wolski, W. 1986. Partikellexikographie. Ein Beitrag zur praktischen Lexikologie. Mit einer englischen Zusammenfassung. Lexicographica Series Maior 14. Tübingen: Niemeyer.

Wolski, W. 1989. Formen der Textverdichtung im allgemeinen einsprachigen Wörterbuch. HSK 5.1: 956-967.

Wolski, W. 1991. Formen der Textverdichtung im zweisprachigen Wörterbuch. HSK 5.3: 2837-2854. 